\title{
Soft contribution to the damping rate of a hard photon in a weakly magnetized hot medium
}

\author{
Ritesh Ghosh, ${ }^{*}$ Bithika Karmakar, ${ }^{\dagger}$ and Munshi G. Mustafa ${ }^{\ddagger}$ \\ Theory Division, Saha Institute of Nuclear Physics, 1/AF, Bidhannagar, Kolkata 700064, India \\ Homi Bhabha National Institute, Anushaktinagar, Mumbai, Maharashtra 400094, India
}

(Received 5 November 2019; accepted 18 February 2020; published 5 March 2020)

\begin{abstract}
We consider weakly magnetized hot QED plasma comprising electrons and positrons. There are three distinct dispersive (longitudinal and two transverse) modes of a photon in a thermomagnetic medium. At lowest order in the coupling constant, a photon is damped in this medium via Compton scattering and pair creation process. We evaluate the damping rate of hard photon by calculating the imaginary part of the each transverse dispersive modes in a thermomagnetic QED medium. We note that one of the fermions in the loop of one-loop photon self-energy is considered as soft and the other one is hard. Considering the resummed fermion propagator in a weakly magnetized medium for the soft fermion and the Schwinger propagator for hard fermion, we calculate the soft contribution to the damping rate of hard photon. In weak field approximation the thermal and thermomagnetic contributions to damping rate get separated out for each transverse dispersive mode. The total damping rate for each dispersive mode in presence of magnetic field is found to be reduced than that of the thermal one. This formalism can easily be extended to QCD plasma.
\end{abstract}

DOI: 10.1103/PhysRevD.101.056007

\section{INTRODUCTION}

Astrophysical plasma is almost always immersed in magnetic field. Extreme, magnetized plasma is found in interiors of neutron star, magnetospheres of magnetars and central engines of supernovae and gamma ray bursts [1]. The propagation of photon through the hot magnetized plasma, viz., electron-positron plasma (EPP), is of great interest. Because the magnetar phenomena are found by analyzing the high-energy radiation detected at earth. Thus it is very important to have a good understanding of the propagation of photon through the EPP. Furthermore, the phenomenon of Faraday rotation i.e., change of polarization of photon while propagating through a medium has been studied in Ref. [2] in a field theoretical viewpoint. This has also been detected in several astrophysical objects [3]. Also high-intensity laser beams are used to create ultrarelativistic EPP of temperature around $10 \mathrm{MeV}$ [4]. This EPP may play an important role in various astrophysical situations. Some properties of such plasma, viz., the equation of state, dispersion relation of collective

*ritesh.ghosh@saha.ac.in

†bithika.karmakar@saha.ac.in

\#munshigolam.mustafa@saha.ac.in

Published by the American Physical Society under the terms of the Creative Commons Attribution 4.0 International license. Further distribution of this work must maintain attribution to the author(s) and the published article's title, journal citation, and DOI. Funded by SCOAP ${ }^{3}$. plasma modes of photon and electron, damping rates, mean free paths, transport coefficients and particle production rates, are studied using QED at finite temperature $[5,6]$.

On the other hand in noncentral heavy ion collisions, the magnetic field as high as $(15-20) m_{\pi}^{2}$ can be generated [7] at LHC energies. After a few $\mathrm{fm} / c$ of the collision, the magnetic field strength ${ }^{1}$ decreases to $(1-2) m_{\pi}^{2}$. The effect of magnetic field on the properties of the QCD matter [viz. quark-gluon plasma (QGP)] and on the phase diagram of QCD is of great interest. Recently, several studies have found the effect of magnetic catalysis [13-16], i.e., the enhancement of phase transition temperature of QCD matter in presence of external magnetic field, whereas, some results of inverse magnetic catalysis [17-25] have been reported. Various properties of QCD matter at weak coupling in presence of magnetic field is being studied including the equation of state [26-28], transport properties [29-31]. Modification of QCD Debye mass and the two point correlation functions of quarks [32] and gluons [33-35] i.e., partons have been analyzed recently. Dilepton production rate from a hot magnetized QCD plasma [12,36-42] has been calculated. The photon is also

\footnotetext{
${ }^{1}$ The initial magnitude of this magnetic field can be very high at the initial time of the heavy-ion collisions and then it decreases very fast, being inversely proportional to the square of time $[8,9]$. However for a different point of view, see Refs. [10-12], where the time dependence of magnetic field is shown to be adiabatic due to the high conductivity of the medium.
} 
considered as a good probe of the QGP medium as photon only interacts electromagnetically. Thus, it comes out of the hot QCD system without interacting much. The damping rate of the hard photon is associated with the mean free path of photon [43] and hard photon production rate in QGP [44].

Damping rate of photon is related to the imaginary part of photon dispersion in the medium [45] which is again related to the scattering cross section of the process that we find by cutting the photon self-energy diagram [46]. In lowest order coupling constant, photons are damped by Compton scattering and pair creation process. In case of low momentum transfer, the damping rate shows infrared singularity. Thus one should consider the effective resummed propagator instead of bare propagator for soft momentum of fermion. We will call this as the soft contribution to the damping rate of photon. The hard contribution refers to the case where all the fermions in loop have momentum order of or much greater than the system temperature $T$. Both soft and hard contributions to the damping rate of hard photon in thermal medium have been calculated in Ref. [45]. The dispersion relations of photon are modified for a hot magnetized medium [33]. So the damping rate of photon will also get modified in a thermomagnetic medium. In this article we intend to compute the soft contribution to the hard photon damping rate for a weakly magnetized hot medium in one loop approximation of photon self-energy. In a thermomagnetic medium this would be a good indicator as higher loop calculation contributing to higher order would be extremely involved.

We consider hard photon of momentum $P^{\mu}=\left(p_{0}, \mathbf{p}\right)$ where $p=|\mathbf{p}| \gg T$ in a relativistic hot magnetized QED medium. To find the soft contribution of the damping rate we introduce a separation scale $\Lambda$ where $e T \ll \Lambda \ll T$ ( $g T \ll \Lambda \ll T$ in case of QCD). In the soft part of the damping rate, the contribution from soft loop momentum involving a fermion is taken into account up to the separation scale $\Lambda$. Here we assume that the magnetic field strength is weak i.e., $\sqrt{e B}<e T<T\left(\sqrt{q_{f} B}<g T<T\right.$ for QCD). We use the recently obtained effective fermion propagator [32] in presence of weak magnetic field for the soft fermion and Schwinger propagator for the hard fermion in the loop. The Braaten-Pisarki-Yuan formalism [47] has been used here to calculate the imaginary part of photon self-energy. Extension to the case of damping rate of hard photon in weakly magnetized hot QCD medium is straightforward. We need to consider the loop fermions as quark and antiquark in that case.

In Sec. II we describe the set up to calculate the photon damping rate associated with imaginary part of photon selfenergy. In Sec. III the self-energy is obtained in a weak field approximation. The imaginary parts of various components of photon self-energy is obtained in Sec. IV. Results are given in Sec. V. We conclude in Sec. VI.

\section{SETUP}

We consider plasma of electrons and positrons at temperature $T$. The $z$-axis of the lab frame is oriented along the magnetic field. The general structure of the gauge boson self-energy and corresponding effective propagator have been evaluated in Ref. [33]. The general covariant structure of photon self-energy in a magnetized hot medium can be written as

$$
\Pi^{\mu \nu}=\beta B^{\mu \nu}+\sigma R^{\mu \nu}+\delta Q^{\mu \nu}+\alpha N^{\mu \nu},
$$

where various form factors can be written as

$$
\begin{aligned}
\beta & =B^{\mu \nu} \Pi_{\mu \nu}, \\
\sigma & =R^{\mu \nu} \Pi_{\mu \nu}, \\
\delta & =Q^{\mu \nu} \Pi_{\mu \nu}, \\
\alpha & =\frac{1}{2} N^{\mu \nu} \Pi_{\mu \nu} .
\end{aligned}
$$

The general covariant structure of photon propagator can be obtained [33] as

$$
\begin{aligned}
D_{\mu \nu}= & \frac{\xi P_{\mu} P_{\nu}}{P^{4}}+\frac{\left(P^{2}-\delta\right) B_{\mu \nu}}{\left(P^{2}-\beta\right)\left(P^{2}-\delta\right)-\alpha^{2}}+\frac{R_{\mu \nu}}{P^{2}-\sigma} \\
& +\frac{\left(P^{2}-\beta\right) Q_{\mu \nu}}{\left(P^{2}-\beta\right)\left(P^{2}-\delta\right)-\alpha^{2}}+\frac{\alpha N_{\mu \nu}}{\left(P^{2}-\beta\right)\left(P^{2}-\delta\right)-\alpha^{2}} .
\end{aligned}
$$

We note that the thermal medium (absence of magnetic field) has two dispersive modes of photon i.e., one degenerate transverse mode and one medium induced plasmon mode due to breaking of boost invariance. Now breaking of rotational invariance in the presence of a magnetic field leads to three dispersive modes of photon by lifting the degeneracy of the transverse modes. These three dispersive modes can be seen from the pole of Eq. (3). Now, the dispersion relations can be written as

$$
\begin{aligned}
& P^{2}-\sigma=0, \\
\left(P^{2}-\delta\right)\left(P^{2}-\beta\right)-\alpha^{2}= & \left(P^{2}-\frac{\beta+\delta+\sqrt{(\beta-\delta)^{2}+4 \alpha^{2}}}{2}\right) \\
& \times\left(P^{2}-\frac{\beta+\delta-\sqrt{(\beta-\delta)^{2}+4 \alpha^{2}}}{2}\right) \\
= & 0 .
\end{aligned}
$$

In the weak magnetic field approximation $\alpha$ does not contribute up to $\mathcal{O}[(e B)]^{2}$, one gets the simple form of the above dispersive modes [26] 


$$
\begin{aligned}
& P^{2}-\sigma=0 \\
& P^{2}-\beta=0 \\
& P^{2}-\delta=0
\end{aligned}
$$

Damping rate is defined as the imaginary part of photon dispersion relation. The medium induced longitudinal (plasmon) mode does not contribute to the damping rate ${ }^{2}$ and the dispersion relations for two transverse modes of a photon are given, respectively, as

$$
P^{2}-\sigma=0, \quad P^{2}-\delta=0,
$$

Damping rates $\gamma_{\delta}(p)$ and $\gamma_{\sigma}(p)$ (for no overdamping i.e., $\gamma_{i} \ll p_{0}$ where $\left.i=\delta, \sigma\right)$ of hard photon are given by imaginary part of the form factors as [48]

$$
\begin{aligned}
& \gamma_{\sigma}(p)=-\frac{1}{2 p} \operatorname{Im} \sigma\left(p_{0}=p\right), \\
& \gamma_{\delta}(p)=-\frac{1}{2 p} \operatorname{Im} \delta\left(p_{0}=p\right) .
\end{aligned}
$$

The tensor structures of $R^{\mu \nu}$ and $Q^{\mu \nu}$ [33] are given as

$$
\begin{aligned}
R^{\mu \nu} & =\left(\begin{array}{cccc}
0 & 0 & 0 & 0 \\
0 & 0 & 0 & 0 \\
0 & 0 & -1 & 0 \\
0 & 0 & 0 & 0
\end{array}\right), \\
Q^{\mu \nu} & =\left(\begin{array}{cccc}
0 & 0 & 0 & 0 \\
0 & -\cos ^{2} \theta_{p} & 0 & \sin \theta_{p} \cos \theta_{p} \\
0 & 0 & 0 & 0 \\
0 & \sin \theta_{p} \cos \theta_{p} & 0 & -\sin ^{2} \theta_{p}
\end{array}\right) .
\end{aligned}
$$

Using Eq. (10) in Eq. (2) we can write the form factors $\sigma$ and $\delta$ in the weak field approximation as

$$
\begin{gathered}
\sigma=-\left(\Pi_{0}^{22}+\Pi_{2}^{22}\right) \\
\delta=-\cos ^{2} \theta_{p}\left(\Pi_{0}^{11}+\Pi_{2}^{11}\right)-\sin ^{2} \theta_{p}\left(\Pi_{0}^{33}+\Pi_{2}^{33}\right) \\
+2 \sin \theta_{p} \cos \theta_{p}\left(\Pi_{0}^{13}+\Pi_{2}^{13}\right) .
\end{gathered}
$$

Combining Eq. (8) with Eq. (11) and Eq. (9) with Eq. (12), the damping rates become

$$
\gamma_{\sigma}(p)=\frac{1}{2 p}\left(\operatorname{Im} \Pi_{0}^{22}+\operatorname{Im} \Pi_{2}^{22}\right)
$$

\footnotetext{
${ }^{2}$ The longitudinal dispersive mode merges with the light cone at high photon momentum.
}

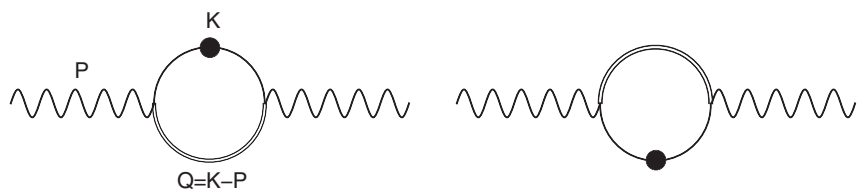

FIG. 1. Photon self-energy where the blob represents the effective electron propagator in magnetic field and double line represents bare electron propagator in magnetic field.

$$
\begin{aligned}
\gamma_{\delta}(p)= & \frac{1}{2 p}\left[\cos ^{2} \theta_{p}\left(\operatorname{Im} \Pi_{0}^{11}+\operatorname{Im} \Pi_{2}^{11}\right)\right. \\
& +\sin ^{2} \theta_{p}\left(\operatorname{Im} \Pi_{0}^{33}+\operatorname{Im} \Pi_{2}^{33}\right) \\
& \left.-2 \sin \theta_{p} \cos \theta_{p}\left(\operatorname{Im} \Pi_{0}^{13}+\operatorname{Im} \Pi_{2}^{13}\right)\right] .
\end{aligned}
$$

The damping rates in Eqs. (13) and (14) can now be written as

$$
\begin{aligned}
& \gamma_{\sigma}(p)=\gamma_{\mathrm{th}}(p)+\gamma_{\sigma}^{B}(p), \\
& \gamma_{\delta}(p)=\gamma_{\mathrm{th}}(p)+\gamma_{\delta}^{B}(p) .
\end{aligned}
$$

where $\gamma_{\text {th }}$ is the $\mathcal{O}\left[(e B)^{0}\right]$ contribution or thermal contribution is given as

$$
\begin{aligned}
\gamma_{\mathrm{th}}(p)= & \frac{1}{2 p} \operatorname{Im} \Pi_{0}^{22}=\frac{1}{2 p}\left[\cos ^{2} \theta_{p} \operatorname{Im} \Pi_{0}^{11}+\sin ^{2} \theta_{p} \operatorname{Im} \Pi_{0}^{33}\right. \\
& \left.-2 \sin \theta_{p} \cos \theta_{p} \operatorname{Im} \Pi_{0}^{13}\right] .
\end{aligned}
$$

The thermomagnetic corrections of $\mathcal{O}\left[(e B)^{2}\right]$ are given as

$$
\begin{gathered}
\gamma_{\sigma}^{B}(p)=\frac{1}{2 p} \operatorname{Im} \Pi_{2}^{22}, \\
\gamma_{\delta}^{B}(p)=\frac{1}{2 p}\left[\cos ^{2} \theta_{p} \operatorname{Im} \Pi_{2}^{11}+\sin ^{2} \theta_{p} \operatorname{Im} \Pi_{2}^{33}\right. \\
\left.-2 \sin \theta_{p} \cos \theta_{p} \operatorname{Im} \Pi_{2}^{13}\right] .
\end{gathered}
$$

We need to obtain the imaginary parts of $11,22,33$, and 13 components of the photon self-energy $\Pi^{\mu \nu}$ which are computed in the following sections.

\section{PHOTON SELF-ENERGY IN HOT MAGNETIZED MEDIUM}

The photon self-energy as shown in Fig. 1 can be written as

$$
\begin{aligned}
\Pi_{\mu \nu}(P)= & i e^{2} \int \frac{d^{4} K}{(2 \pi)^{4}}\left\{\operatorname{Tr}\left[\gamma_{\mu} S^{*}(K) \gamma_{\nu} S(Q)\right]\right. \\
& \left.+\operatorname{Tr}\left[\gamma_{\nu} S^{*}(K) \gamma_{\mu} S(Q)\right]\right\},
\end{aligned}
$$

where $S^{*}(K)$ is effective electron propagator and $S(K)$ is Schwinger propagator for bare electron. As the external 
photon is hard, we consider one bare and one effective fermion propagator in the loop. In the following we would obtain the propagators for fermion.

\section{A. Fermion propagator in weak field approximation}

In the weak magnetic field limit, i.e., $\sqrt{e B}<m_{\text {th }} \sim$ $e T<T$ the Schwinger propagator for fermion can be written up to $\mathcal{O}\left[(e B)^{2}\right]$ as [49]

$$
\begin{aligned}
S(K)= & \frac{K+m_{f}}{K^{2}-m_{f}^{2}}+i \gamma^{1} \gamma^{2} \frac{K_{\|}+m_{f}}{\left(K^{2}-m_{f}^{2}\right)^{2}}(e B) \\
& +2\left[\frac{\{(K \cdot u) \not h-(K \cdot n) \not h\}-\not K}{\left(K^{2}-m_{f}^{2}\right)^{3}}-\frac{k_{\perp}^{2}\left(K+m_{f}\right)}{\left(K^{2}-m_{f}^{2}\right)^{4}}\right](e B)^{2} \\
& +\mathcal{O}\left[(e B)^{3}\right] \\
= & S_{0}+S_{1}+S_{2}+\mathcal{O}\left[(e B)^{3}\right] .
\end{aligned}
$$

The general form of fermion self-energy in a weakly magnetized medium can be written as [32]

$$
\Sigma(K)=-a K K-b \not h-b^{\prime} \gamma_{5} \not h-c^{\prime} \gamma_{5} \not h .
$$

In one loop order, the form factors are given as

$$
\begin{gathered}
a\left(k_{0}, k\right)=-\frac{m_{\mathrm{th}}^{2}}{k^{2}} Q_{1}\left(\frac{k_{0}}{k}\right), \\
b\left(k_{0}, k\right)=\frac{m_{\mathrm{th}}^{2}}{k}\left[\frac{k_{0}}{k} Q_{1}\left(\frac{k_{0}}{k}\right)-Q_{0}\left(\frac{k_{0}}{k}\right)\right], \\
b^{\prime}\left(k_{0}, k\right)=4 e^{2} M^{2}\left(T, m_{f}, e B\right) \frac{k_{3}}{k^{2}} Q_{1}\left(\frac{k_{0}}{k}\right), \\
c^{\prime}\left(k_{0}, k\right)=4 e^{2} M^{2}\left(T, m_{f}, e B\right) \frac{1}{k} Q_{0}\left(\frac{k_{0}}{k}\right),
\end{gathered}
$$

where Legendre function of second kind are given as

$$
Q_{0}(x)=\frac{1}{2} \ln \left(\frac{x+1}{x-1}\right),
$$

$$
Q_{1}(x)=x Q_{0}(x)-1,
$$

and the thermomagnetic mass is given as

$$
M^{2}\left(T, m_{f}, e B\right)=\frac{e B}{16 \pi^{2}}\left[\ln 2-\frac{\pi T}{2 m_{f}}\right],
$$

whereas thermal mass is given as

$$
m_{\mathrm{th}}^{2}=\frac{1}{8} e^{2} T^{2} .
$$

The effective fermion propagator can be written [32] as

$$
\begin{aligned}
S^{*}(K) & =P_{-} \frac{\not L(K)}{L^{2}} P_{+}+P_{+} \frac{\not R(K)}{R^{2}} P_{-} \\
& =S_{L}^{*}(K)+S_{R}^{*}(K),
\end{aligned}
$$

where chirality projection operators are given by

$$
P_{ \pm}=\frac{1}{2}\left(1 \pm \gamma_{5}\right)
$$

and $L^{\mu}$ and $R^{\mu}$ are given as

$$
\begin{aligned}
& L^{\mu}=(1+a) K^{\mu}+\left(b+b^{\prime}\right) u^{\mu}+c^{\prime} n^{\mu}, \\
& R^{\mu}=(1+a) K^{\mu}+\left(b-b^{\prime}\right) u^{\mu}-c^{\prime} n^{\mu} .
\end{aligned}
$$

For simplicity of calculation we expand the effective fermion propagator in Eq. (31) in powers of $e B$ and keep terms up to $\mathcal{O}\left[(e B)^{2}\right]$ as

$$
S^{*}(K)=S_{0}^{*}(K)+S_{1}^{*}(K)+S_{2}^{*}(K)+\mathcal{O}\left[(e B)^{3}\right],
$$

where $S_{0}^{*}(K)$ is $\mathcal{O}\left[(e B)^{0}\right]$ and given as

$$
S_{0}^{*}(K)=\frac{(1+a) K+b \not h}{D^{2}}=\frac{(1+a) K+b \not h}{D_{+} D_{-}},
$$

where $D_{ \pm}=(1+a)\left(k_{0} \mp k\right)+b$.

Equation (36) is the effective HTL fermion propagator $[50,51]$ in thermal medium. The $\mathcal{O}[(e B)]$ is obtained as

$$
\begin{aligned}
S_{1}^{*}(K)= & \frac{1}{D^{4}}\left[2(1+a) K \gamma_{5}\left\{-(1+a) k_{3} c^{\prime}-(1+a) k_{0} b^{\prime}-b b^{\prime}\right\}+\not h \gamma_{5}\left\{\left((1+a)^{2} K^{2}-b^{2}\right) b^{\prime}-2(a+1) b c^{\prime} k_{3}\right\}\right. \\
& \left.+c^{\prime} \not h \gamma_{5}\left\{\left(2(1+a) k_{0}+b\right) b+(a+1)^{2} K^{2}\right\}\right],
\end{aligned}
$$

whereas $\mathcal{O}\left[(e B)^{2}\right]$ is obtained as

$$
\begin{aligned}
S_{2}^{*}(K) & =\left[\frac{\left(2 b^{\prime}\left\{(1+a) k_{0}+b\right\}+2 c^{\prime} k_{3}(1+a)\right)^{2}}{D^{6}}-\frac{b^{\prime 2}-c^{\prime 2}}{D^{4}}\right]\{(1+a) K+b \not h\}-\frac{\left(2 b^{\prime}\left\{(1+a) k_{0}+b\right\}+2 c^{\prime} k_{3}(1+a)\right)\left(b^{\prime} \not h+c^{\prime} \not h\right)}{D^{4}} \\
& =\left(\frac{h^{2}\left(k_{0}, k_{\perp}, k_{3}\right)}{D^{6}}-\frac{h^{\prime}}{D^{4}}\right)\{(1+a) K+b \not h\}-\frac{h\left(k_{0}, k_{\perp}, k_{3}\right)}{D^{4}}\left(b^{\prime} \not h+c^{\prime} \not h\right),
\end{aligned}
$$


where $h=2 b^{\prime}\left\{(1+a) k_{0}+b\right\}+2 c^{\prime} k_{3}(1+a)$ and $h^{\prime}=b^{\prime 2}-c^{\prime 2}$.

\section{B. Photon self-energy in weak magnetic field}

Now the $\mathcal{O}\left[(e B)^{0}\right]$ contribution of $\Pi^{\mu \nu}$ given in Eq. (20) can be written as

$$
\begin{aligned}
\Pi_{0}^{\mu \nu} & =i e^{2} \int \frac{d^{4} K}{(2 \pi)^{4}}\left\{\operatorname{Tr}\left[\gamma_{\mu} S_{0}^{*}(K) \gamma_{\nu} S_{0}(Q)\right]+\operatorname{Tr}\left[\gamma_{\nu} S_{0}^{*}(K) \gamma_{\mu} S_{0}(Q)\right]\right\} \\
& =i e^{2} \int \frac{d^{4} K}{(2 \pi)^{4}}\left\{\operatorname{Tr}\left[\gamma^{\mu}\left(\frac{\gamma_{0}-\vec{\gamma} \cdot \hat{k}}{2 D_{+}}+\frac{\gamma_{0}+\vec{\gamma} \cdot \hat{k}}{2 D_{-}}\right) \gamma^{\nu}\left(f_{0}^{(1)} \gamma^{0}-f_{0}^{(0)} \vec{\gamma} \cdot \vec{q}\right)\right]+\operatorname{Tr}\left[\gamma^{\nu}\left(\frac{\gamma_{0}-\vec{\gamma} \cdot \hat{k}}{2 D_{+}}+\frac{\gamma_{0}+\vec{\gamma} \cdot \hat{k}}{2 D_{-}}\right) \gamma^{\mu}\left(f_{0}^{(1)} \gamma^{0}-f_{0}^{(0)} \vec{\gamma} \cdot \vec{q}\right)\right]\right\} \\
& =8 i e^{2} \int \frac{d^{4} K}{(2 \pi)^{4}} \frac{(1+a)\left\{\left(K^{\mu} Q^{\nu}+K^{\nu} Q^{\mu}\right)-g^{\mu \nu} K \cdot Q\right\}+b\left\{\left(Q^{\mu} u^{\nu}+Q^{\nu} u^{\mu}\right)-g^{\mu \nu} Q \cdot u\right\}}{D_{+} D_{-} Q^{2}}
\end{aligned}
$$

where

$$
f_{0}^{(1)}=\frac{q_{0}}{Q^{2}}, \quad f_{0}^{(0)}=\frac{1}{Q^{2}}, \quad f_{1}^{(1)}=\frac{q_{0}}{Q^{4}}, \quad f_{1}^{(0)}=\frac{1}{Q^{4}}
$$

The $\mathcal{O}[(e B)]$ contribution of $\Pi^{\mu \nu}$ is given as

$\Pi_{1}^{\mu \nu}=i e^{2} \int \frac{d^{4} K}{(2 \pi)^{4}}\left\{\operatorname{Tr}\left[\gamma_{\mu} S_{0}^{*}(K) \gamma_{\nu} S_{1}(Q)\right]+\operatorname{Tr}\left[\gamma_{\nu} S_{0}^{*}(K) \gamma_{\mu} S_{1}(Q)\right]+\operatorname{Tr}\left[\gamma_{\mu} S_{1}^{*}(K) \gamma_{\nu} S_{0}(Q)\right]+\operatorname{Tr}\left[\gamma_{\nu} S_{1}^{*}(K) \gamma_{\mu} S_{0}(Q)\right]\right\}$,

which becomes zero.

The $\mathcal{O}\left[(e B)^{2}\right]$ contribution of $\Pi^{\mu \nu}$ is given as

$$
\begin{aligned}
\Pi_{2}^{\mu \nu}= & i e^{2} \int \frac{d^{4} K}{(2 \pi)^{4}}\left\{\operatorname{Tr}\left[\gamma_{\mu} S_{1}^{*}(K) \gamma_{\nu} S_{1}(Q)\right]+\operatorname{Tr}\left[\gamma_{\nu} S_{1}^{*}(K) \gamma_{\mu} S_{1}(Q)\right]+\operatorname{Tr}\left[\gamma_{\mu} S_{0}^{*}(K) \gamma_{\nu} S_{2}(Q)\right]\right. \\
& \left.+\operatorname{Tr}\left[\gamma_{\nu} S_{0}^{*}(K) \gamma_{\mu} S_{2}(Q)\right]+\operatorname{Tr}\left[\gamma_{\mu} S_{2}^{*}(K) \gamma_{\nu} S_{0}(Q)\right]+\operatorname{Tr}\left[\gamma_{\nu} S_{2}^{*}(K) \gamma_{\mu} S_{0}(Q)\right]\right\}
\end{aligned}
$$

We calculate the above mentioned trace as follows. The trace of the first and second terms of Eq. (42) can be calculated as

$$
\begin{aligned}
\operatorname{Tr}\left[\gamma_{\mu} S_{1}^{*}(K) \gamma_{\nu} S_{1}(Q)\right]+\operatorname{Tr}\left[\gamma_{\nu} S_{1}^{*}(K) \gamma_{\mu} S_{1}(Q)\right]= & \frac{8(e B)}{D^{2}\left(Q^{2}-m_{f}^{2}\right)^{2}}\left[b^{\prime}\left\{\left(u^{\mu} n^{\nu}+u^{\nu} n^{\mu}\right)(Q \cdot u)-2 u^{\mu} u^{\nu}(Q \cdot n)+g^{\mu \nu}(Q \cdot n)\right\}\right. \\
& \left.+c^{\prime}\left\{2 n^{\mu} n^{\nu}(Q \cdot u)-\left(n^{\mu} u^{\nu}+n^{\nu} u^{\mu}\right)(Q \cdot n)+g^{\mu \nu}(Q \cdot u)\right\}\right] \\
& -\frac{8(e B)}{D^{4}\left(Q^{2}-m_{f}^{2}\right)^{2}}\left[h \left\{( 1 + a ) \left(g^{\mu \nu}((K \cdot u)(Q \cdot n)-(K \cdot n)(Q \cdot u))\right.\right.\right. \\
& \left.-\left(K^{\mu} u^{\nu}+K^{\nu} u^{\mu}\right) Q \cdot n+\left(K^{\mu} n^{\nu}+K^{\nu} n^{\mu}\right) Q \cdot u\right)+b\left(g^{\mu \nu} Q \cdot n\right. \\
& \left.\left.\left.+\left(u^{\mu} n^{\nu}+u^{\nu} n^{\mu}\right) Q \cdot u-2 u^{\mu} u^{\nu} Q \cdot n\right)\right\}\right] .
\end{aligned}
$$

The trace of third and fourth terms in Eq. (42) can be obtained as

$$
\begin{aligned}
\operatorname{Tr}\left[\gamma_{\mu} S_{0}^{*}(K) \gamma_{\nu} S_{2}(Q)\right]+\operatorname{Tr}\left[\gamma_{\nu} S_{0}^{*}(K) \gamma_{\mu} S_{2}(Q)\right]= & \frac{8(e B)^{2}}{D_{+}\left(Q^{2}-m_{f}^{2}\right)^{3}}\left[q_{0}\left(\hat{K}^{\mu} g^{0 \nu}+\hat{K}^{\nu} g^{0 \mu}-g^{\mu \nu}\right)-q_{3}\left(\hat{K}^{\mu} g^{3 \nu}+\hat{K}^{\nu} g^{3 \mu}-g^{\mu \nu} \hat{k}_{3}\right)\right. \\
& \left.-\left(\hat{K}^{\mu} Q^{\nu}+\hat{K}^{\nu} Q^{\mu}-g^{\mu \nu} \hat{K} \cdot Q\right)\right]-\frac{8(e B)^{2} q_{\perp}^{2}}{D_{+}\left(Q^{2}-m_{f}^{2}\right)^{4}}\left[\hat{K}^{\mu} Q^{\nu}+\hat{K}^{\nu} Q^{\mu}-g^{\mu \nu} \hat{K}^{\prime} \cdot Q\right] \\
& +\frac{8(e B)^{2}}{D_{-}\left(Q^{2}-m_{f}^{2}\right)^{3}}\left[q_{0}\left(\hat{K}^{\prime \mu} g^{0 \nu}+\hat{K}^{\prime \nu} g^{0 \mu}-g^{\mu \nu}\right)-q_{3}\left(\hat{K}^{\prime \mu} g^{3 \nu}+\hat{K}^{\prime \nu} g^{3 \mu}+g^{\mu \nu} \hat{k}_{3}\right)\right. \\
& \left.-\left(\hat{K}^{\prime \mu} Q^{\nu}+\hat{K}^{\prime \nu} Q^{\mu}-g^{\mu \nu} \hat{K}^{\prime} \cdot Q\right)\right]-\frac{8(e B)^{2} q_{\perp}^{2}}{D_{-}\left(Q^{2}-m_{f}^{2}\right)^{4}}\left[\hat{K}^{\prime \mu} Q^{\nu}+\hat{K}^{\prime \nu} Q^{\mu}-g^{\mu \nu} \hat{K}^{\prime} \cdot Q\right],
\end{aligned}
$$


where $\hat{K}^{\prime \mu}=(1,-\hat{\mathbf{k}})$. The trace of fifth and sixth terms in Eq. (42) are obtained as

$$
\begin{aligned}
\operatorname{Tr}\left[\gamma_{\mu} S_{2}^{*}(K) \gamma_{\nu} S_{0}(Q)\right]+\operatorname{Tr}\left[\gamma_{\nu} S_{2}^{*}(K) \gamma_{\mu} S_{0}(Q)\right] \\
=\frac{8}{\left(Q^{2}-m_{f}^{2}\right)}\left[\left(\frac{h^{2}}{D^{6}}-\frac{h^{\prime}}{D^{4}}\right)(1+a)\left(K^{\mu} Q^{\nu}+K^{\nu} Q^{\mu}-g^{\mu \nu} K \cdot Q\right)+\left(b\left(\frac{h^{2}}{D^{6}}-\frac{h^{\prime}}{D^{4}}\right)-b^{\prime} \frac{h}{D^{4}}\right)\right. \\
\left.\quad \times\left(g^{\mu 0} Q^{\nu}+g^{\nu 0} Q^{\mu}-g^{\mu \nu} q_{0}\right)-c^{\prime} \frac{h}{D^{4}}\left(g^{\mu 3} Q^{\nu}+g^{\nu 3} Q^{\mu}-g^{\mu \nu} q_{3}\right)\right] .
\end{aligned}
$$

The photon self-energy in weak field approximation now can be decomposed using Eqs. (39), (41), (42) as

$$
\Pi^{\mu \nu}(P)=\Pi_{0}^{\mu \nu}(P)+\Pi_{2}^{\mu \nu}(P)
$$

where the first term is a pure thermal $\left(\mathcal{O}\left[(e B)^{0}\right]\right)$ contribution and second term is thermomagnetic correction of $\mathcal{O}\left[(e B)^{2}\right]$.

Now the $\mathcal{O}\left[(e B)^{0}\right]$ expression of $\Pi^{11}, \Pi^{22}, \Pi^{33}$, and $\Pi^{13}$ can be written from Eq. (39) as

$$
\begin{aligned}
& \Pi_{0}^{11}\left(p_{0}, p\right)=8 i e^{2} \int \frac{d^{4} K}{(2 \pi)^{4}} \frac{(1+a)\left(k_{0} q_{0}+2 k_{1} q_{1}-\vec{k} \cdot \vec{q}\right)+b q_{0}}{\left\{\left((1+a) k_{0}+b\right)^{2}-(1+a)^{2} k^{2}\right\} Q^{2}} \\
& =-4 e^{2} \mathcal{Y}\left[\left(\frac{f_{0}^{(1)}}{D_{+}}+\frac{f_{0}^{(1)}}{D_{-}}\right)+\left(2 \hat{k}_{1} q_{1}-\hat{k} \cdot q\right)\left\{\frac{f_{0}^{(0)}}{D_{+}}-\frac{f_{0}^{(0)}}{D_{-}}\right\}\right], \\
& \Pi_{0}^{22}\left(p_{0}, p\right)=8 i e^{2} \int \frac{d^{4} K}{(2 \pi)^{4}} \frac{(1+a)\left(k_{0} q_{0}+2 k_{2} q_{2}-\vec{k} \cdot \vec{q}\right)+b q_{0}}{\left\{\left((1+a) k_{0}+b\right)^{2}-(1+a)^{2} k^{2}\right\} Q^{2}} \\
& =-4 e^{2} y\left[\left(\frac{f_{0}^{(1)}}{D_{+}}+\frac{f_{0}^{(1)}}{D_{-}}\right)+\left(2 \hat{k}_{2} q_{2}-\hat{k} \cdot q\right)\left\{\frac{f_{0}^{(0)}}{D_{+}}-\frac{f_{0}^{(0)}}{D_{-}}\right\}\right] \text {, } \\
& \Pi_{0}^{33}\left(p_{0}, p\right)=8 i e^{2} \int \frac{d^{4} K}{(2 \pi)^{4}} \frac{(1+a)\left(k_{0} q_{0}+2 k_{3} q_{3}-\vec{k} \cdot \vec{q}\right)+b q_{0}}{\left\{\left((1+a) k_{0}+b\right)^{2}-(1+a)^{2} k^{2}\right\} Q^{2}} \\
& =-4 e^{2} \mathcal{Y}\left[\left(\frac{f_{0}^{(1)}}{D_{+}}+\frac{f_{0}^{(1)}}{D_{-}}\right)-\left(\hat{k} \cdot q-2 \hat{k}_{3} q_{3}\right)\left\{\frac{f_{0}^{(0)}}{D_{+}}-\frac{f_{0}^{(0)}}{D_{-}}\right\}\right],
\end{aligned}
$$

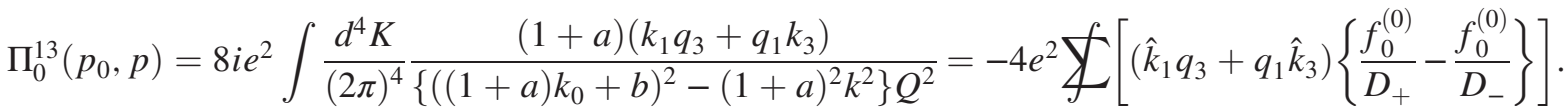

Using Eqs. (42), (43), (44), and (45), one can write the $\mathcal{O}\left[(e B)^{2}\right]$ expression of $\Pi^{11}, \Pi^{22}, \Pi^{33}$, and $\Pi^{13}$ as

$$
\begin{aligned}
\Pi_{2}^{11}= & -e^{2} Y\left[-\frac{8 e B}{D^{2}\left(Q^{2}-m_{f}^{2}\right)^{2}}\left\{b^{\prime} q_{3}+c^{\prime} q_{0}\right\}+\frac{8 e B}{D^{4}\left(Q^{2}-m_{f}^{2}\right)^{2}} h\left\{(1+a)\left(k_{0} q_{3}-k_{3} q_{0}\right)+b q_{3}\right\}\right. \\
& +\frac{8(e B)^{2}}{D_{+}\left(Q^{2}-m_{f}^{2}\right)^{3}}\left(\hat{k}_{2} q_{2}-\hat{k}_{1} q_{1}\right)-\frac{8(e B)^{2} q_{\perp}^{2}}{D_{+}\left(Q^{2}-m_{f}^{2}\right)^{4}}\left(q_{0}-\hat{k} \cdot q+2 \hat{k}_{1} q_{1}\right)-\frac{8(e B)^{2}}{D_{-}\left(Q^{2}-m_{f}^{2}\right)^{3}}\left(\hat{k}_{2} q_{2}-\hat{k}_{1} q_{1}\right) \\
& -\frac{8(e B)^{2} q_{\perp}^{2}}{D_{-}\left(Q^{2}-m_{f}^{2}\right)^{4}}\left(q_{0}+\hat{k} \cdot q-2 \hat{k}_{1} q_{1}\right) \\
& \left.+\frac{8}{\left(Q^{2}-m_{f}^{2}\right)}\left\{\left(\frac{h^{2}}{D^{6}}-\frac{h^{\prime}}{D^{4}}\right)(1+a)\left(2 k_{1} q_{1}+K \cdot Q\right)+\left(b\left(\frac{h^{2}}{D^{6}}-\frac{h^{\prime}}{D^{4}}\right)-\frac{b^{\prime} h}{D^{4}}\right) q_{0}-\frac{c^{\prime} h}{D^{4}} q_{3}\right\}\right],
\end{aligned}
$$




$$
\begin{aligned}
& \Pi_{2}^{22}=-e^{2} y\left[-\frac{8 e B}{D^{2}\left(Q^{2}-m_{f}^{2}\right)^{2}}\left\{b^{\prime} q_{3}+c^{\prime} q_{0}\right\}+\frac{8 e B}{D^{4}\left(Q^{2}-m_{f}^{2}\right)^{2}} h\left\{(1+a)\left(k_{0} q_{3}-k_{3} q_{0}\right)+b q_{3}\right\}\right. \\
& +\frac{8(e B)^{2}}{D_{+}\left(Q^{2}-m_{f}^{2}\right)^{3}}\left(\hat{k}_{1} q_{1}-\hat{k}_{2} q_{2}\right)-\frac{8(e B)^{2} q_{\perp}^{2}}{D_{+}\left(Q^{2}-m_{f}^{2}\right)^{4}}\left(q_{0}-\hat{k} \cdot q+2 \hat{k}_{2} q_{2}\right)-\frac{8(e B)^{2}}{D_{-}\left(Q^{2}-m_{f}^{2}\right)^{3}}\left(\hat{k}_{1} q_{1}-\hat{k}_{2} q_{2}\right) \\
& -\frac{8(e B)^{2} q_{\perp}^{2}}{D_{-}\left(Q^{2}-m_{f}^{2}\right)^{4}}\left(q_{0}+\hat{k} \cdot q-2 \hat{k}_{2} q_{2}\right)+\frac{8}{\left(Q^{2}-m_{f}^{2}\right)}\left\{\left(\frac{h^{2}}{D^{6}}-\frac{h^{\prime}}{D^{4}}\right)(1+a)\left(2 k_{2} q_{2}+K \cdot Q\right)\right. \\
& \left.\left.+\left(b\left(\frac{h^{2}}{D^{6}}-\frac{h^{\prime}}{D^{4}}\right)-\frac{b^{\prime} h}{D^{4}}\right) q_{0}-\frac{c^{\prime} h}{D^{4}} q_{3}\right\}\right] \\
& \Pi_{2}^{33}=-e^{2} Y\left[\frac{8 e B}{D^{2}\left(Q^{2}-m_{f}^{2}\right)^{2}}\left\{-b^{\prime} q_{3}+c^{\prime} q_{0}\right\}+\frac{8 e B}{D^{4}\left(Q^{2}-m_{f}^{2}\right)^{2}} h\left\{(1+a)\left(k_{0} q_{3}+k_{3} q_{0}\right)+b q_{3}\right\}\right. \\
& +\frac{8(e B)^{2}}{D_{+}\left(Q^{2}-m_{f}^{2}\right)^{3}}\left(-q_{3} \hat{k}_{3}+\hat{k} \cdot q\right)-\frac{8(e B)^{2} q_{\perp}^{2}}{D_{+}\left(Q^{2}-m_{f}^{2}\right)^{4}}\left(q_{0}-\hat{k} \cdot q+2 q_{3} \hat{k}_{3}\right) \\
& +\frac{8(e B)^{2}}{D_{-}\left(Q^{2}-m_{f}^{2}\right)^{3}}\left(q_{3} \hat{k}_{3}-\hat{k} \cdot q\right)-\frac{8(e B)^{2} q_{\perp}^{2}}{D_{-}\left(Q^{2}-m_{f}^{2}\right)^{4}}\left(q_{0}+\hat{k} \cdot q-2 q_{3} \hat{k}_{3}\right) \\
& \left.+\frac{8}{\left(Q^{2}-m_{f}^{2}\right)}\left\{\left(\frac{h^{2}}{D^{6}}-\frac{h^{\prime}}{D^{4}}\right)(1+a)\left(2 k_{3} q_{3}+K \cdot Q\right)+\left(b\left(\frac{h^{2}}{D^{6}}-\frac{h^{\prime}}{D^{4}}\right)-\frac{b^{\prime} h}{D^{4}}\right) q_{0}+\frac{c^{\prime} h}{D^{4}} q_{3}\right\}\right], \\
& \Pi_{2}^{13}=-e^{2} y\left[\frac{8 e B}{D^{4}\left(Q^{2}-m_{f}^{2}\right)^{2}} h(1+a)\left(k_{1} q_{0}\right)-\frac{8(e B)^{2}}{D_{+}\left(Q^{2}-m_{f}^{2}\right)^{3}} \hat{k}_{3} q_{1}-\frac{8(e B)^{2} q_{\perp}^{2}}{D_{+}\left(Q^{2}-m_{f}^{2}\right)^{4}}\right. \\
& \times\left(\hat{k}_{1} q_{3}+\hat{k}_{3} q_{1}\right)+\frac{8(e B)^{2}}{D_{-}\left(Q^{2}-m_{f}^{2}\right)^{3}} \hat{k}_{3} q_{1}+\frac{8(e B)^{2} q_{\perp}^{2}}{D_{-}\left(Q^{2}-m_{f}^{2}\right)^{4}}\left(\hat{k}_{1} q_{3}+\hat{k}_{3} q_{1}\right)+\frac{8}{\left(Q^{2}-m_{f}^{2}\right)} \\
& \left.\times\left\{\left(\frac{h^{2}}{D^{6}}-\frac{h^{\prime}}{D^{4}}\right)(1+a)\left(k_{1} q_{3}+k_{3} q_{1}\right)+\frac{c^{\prime} h}{D^{4}} q_{1}\right\}\right] \text {. }
\end{aligned}
$$

\section{IMAGINARY PARTS OF THE COMPONENTS OF THE PHOTON SELF-ENERGY}

Before obtaining the imaginary parts, we discuss below the various approximations used in this calculation.

(1) We have considered the momentum of photon as hard $(p \gg T)$. The momentum of soft fermion $k \ll T$. Thus we can take the following approximations:

$$
n_{F}(\omega) \sim 1, \quad n_{F}(p-\omega) \sim e^{-p / T}, \quad e^{-p / T} \sim 0 .
$$

(2) An upper cutoff $\Lambda(<T)$ of the soft fermion momentum $k$ has been introduced in the integrations.

(3) We consider $m_{f}=m_{\text {th }}$ for electron.

(4) To perform the various integrations we choose a frame of reference as shown in Fig. 2 in which the external momentum of the photon in $x z$ plane with $0<\theta_{p}<\pi / 2$. So one can write

$$
\vec{p} \equiv\left(p \sin \theta_{p}, 0, p \cos \theta_{p}\right),
$$

and then the loop momentum as

$$
\vec{k} \equiv(k \sin \theta \cos \phi, k \sin \theta \sin \phi, k \cos \theta)
$$

In the following subsection we will obtain imaginary parts of various self-energy components.

\section{A. Imaginary parts of the magnetic field independent part, i.e., $\mathcal{O}\left[(e B)^{0}\right]$}

We evaluate the imaginary parts of $\Pi_{0}^{11}, \Pi_{0}^{22}, \Pi_{0}^{33}$, and $\Pi_{0}^{13}$ using the Braaten-Pisarski-Yuan method $[45,47]$.

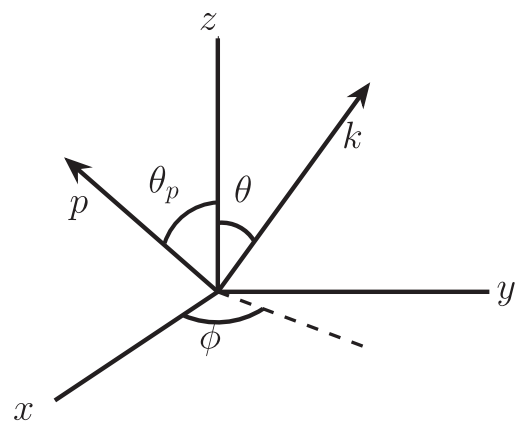

FIG. 2. Choice of reference frame for computing the various components of photon self-energy. The magnetic field is along $\mathrm{z}-$ direction and $\theta_{p}$ is the angle between momentum of photon and the external magnetic field. 


$$
\begin{aligned}
& \operatorname{Im} \Pi_{0}^{11}=-4 e^{2} \pi\left(1-e^{p / T}\right) \int \frac{d^{3} k}{(2 \pi)^{3}} \int_{-\infty}^{\infty} \int_{-\infty}^{\infty} d \omega d \omega^{\prime} n_{F}(\omega) n_{F}\left(\omega^{\prime}\right)\left\{\rho_{0}^{(1)}\left(\omega^{\prime}\right)\left(\rho_{D_{+}}(\omega)+\rho_{D_{-}}(\omega)\right)\right. \\
&\left.-\left(\hat{k} \cdot q-2 \hat{k}_{1} q_{1}\right)\left(\rho_{D_{+}}(\omega)-\rho_{D_{-}}(\omega)\right) \rho_{0}^{(0)}\left(\omega^{\prime}\right)\right\} \delta\left(\omega+\omega^{\prime}-p\right), \\
& \operatorname{Im} \Pi_{0}^{22}=-4 e^{2} \pi\left(1-e^{p / T}\right) \int \frac{d^{3} k}{(2 \pi)^{3}} \int_{-\infty}^{\infty} \int_{-\infty}^{\infty} d \omega d \omega^{\prime} n_{F}(\omega) n_{F}\left(\omega^{\prime}\right)\left\{\rho_{0}^{(1)}\left(\omega^{\prime}\right)\left(\rho_{D_{+}}(\omega)+\rho_{D_{-}}(\omega)\right)\right. \\
&\left.-\left(\hat{k} \cdot q-2 \hat{k}_{2} q_{2}\right)\left(\rho_{D_{+}}(\omega)-\rho_{D_{-}}(\omega)\right) \rho_{0}^{(0)}\left(\omega^{\prime}\right)\right\} \delta\left(\omega+\omega^{\prime}-p\right), \\
& \operatorname{Im} \Pi_{0}^{33}=-4 e^{2} \pi\left(1-e^{p / T}\right) \int \frac{d^{3} k}{(2 \pi)^{3}} \int_{-\infty}^{\infty} \int_{-\infty}^{\infty} d \omega d \omega^{\prime} n_{F}(\omega) n_{F}\left(\omega^{\prime}\right)\left\{\rho_{0}^{(1)}\left(\omega^{\prime}\right)\left(\rho_{D_{+}}(\omega)+\rho_{D_{-}}(\omega)\right)\right. \\
&\left.-\left(\hat{k} \cdot q-2 \hat{k}_{3} q_{3}\right)\left(\rho_{D_{+}}(\omega)-\rho_{D_{-}}(\omega)\right) \rho_{0}^{(0)}\left(\omega^{\prime}\right)\right\} \delta\left(\omega+\omega^{\prime}-p\right), \\
& \operatorname{Im} \Pi_{0}^{13}=-4 e^{2} \pi\left(1-e^{p / T}\right) \int \frac{d^{3} k}{(2 \pi)^{3}} \int_{-\infty}^{\infty} \int_{-\infty}^{\infty} d \omega d \omega^{\prime} n_{F}(\omega) n_{F}\left(\omega^{\prime}\right)\left\{\left(\hat{k}_{1} q_{3}+q_{1} \hat{k}_{3}\right)\left(\rho_{D_{+}}(\omega)-\rho_{D_{-}}(\omega)\right) \rho_{0}^{(0)}\left(\omega^{\prime}\right)\right\} \delta\left(\omega+\omega^{\prime}-p\right),
\end{aligned}
$$

where $\rho_{0}^{(0)}, \rho_{0}^{(1)}, \rho_{1}^{(0)}, \rho_{1}^{(1)}, \rho_{D_{+}}$, and $\rho_{D_{-}}$are spectral representation of $f_{0}^{(0)}, f_{0}^{(1)}, f_{1}^{(0)}, f_{1}^{(1)}, 1 / D_{+}$, and $1 / D_{-}$respectively. These spectral functions are obtained in Appendix. We know that both $\rho_{D_{+}}$and $\rho_{D_{-}}$have pole containing the mass shell $\delta$-function + Landau cut part in space like region whereas $\rho_{0}^{(0)}, \rho_{0}^{(1)}, \rho_{1}^{(0)}, \rho_{1}^{(1)}$ have only pole containing the mass shell $\delta$ function. Since imaginary parts of various components of the self-energy contain the product of two spectral functions, it would then have the pole-pole and the pole-cut contributions.

The pole-pole parts of $\operatorname{Im} \Pi_{11}, \operatorname{Im}_{22}, \operatorname{Im}_{33}$ and $\operatorname{Im}_{13}$ contain $\delta\left(p-\omega_{ \pm}-q\right)$ where $\omega_{ \pm}$is the energy of the fermion quasiparticle, $\vec{k}$ and $\vec{q}=\vec{k}-\vec{p}$ are the momenta of soft and hard fermion, respectively. Hence $\omega_{ \pm}>k$. The $\delta$ function yields

$$
\begin{aligned}
p-\omega_{ \pm}-q & =0 \\
\cos \phi & \approx \frac{\omega_{ \pm} / k-\cos \theta \cos \theta_{p}}{\sin \theta \sin \theta_{p}} .
\end{aligned}
$$

The value of $\frac{\omega_{ \pm} / k-\cos \theta \cos \theta_{p}}{\sin \theta \sin \theta_{p}}$ excludes the range $[-1,1]$ for all values of the parameters $\theta$ and $\theta_{p}$. This restriction is valid for both thermal and the magnetic case. Thus pole-pole parts do not contribute in this calculation $[44,45]$. In $\mathcal{O}\left[(e B)^{0}\right]$ the contribution comes only from the polecut part.

\section{Pole-cut part of $\mathcal{O}\left[(e B)^{0}\right]$}

Now we would find the pole-cut part of the above selfenergy components in Eqs. (55), (56), (57), and (58) as

$$
\begin{aligned}
\left.\operatorname{Im} \Pi_{0}^{11}\right|_{\text {pole-cut }}= & 2 e^{2} \pi\left(1-e^{p / T}\right) \int \frac{d^{3} k}{(2 \pi)^{3}} \int_{-\infty}^{\infty} \int_{-\infty}^{\infty} d \omega d \omega^{\prime} n_{F}(\omega) n_{F}\left(\omega^{\prime}\right)\left\{\delta\left(\omega^{\prime}-q\right) \Theta\left(k^{2}-\omega^{2}\right) \times\left(\beta_{+}(\omega)+\beta_{-}(\omega)\right)\right. \\
& \left.-\frac{1}{q}\left(\hat{k} \cdot q-2 \hat{k}_{1} q_{1}\right) \delta\left(\omega^{\prime}-q\right) \Theta\left(k^{2}-\omega^{2}\right)\left(\beta_{+}(\omega)-\beta_{-}(\omega)\right)\right\} \delta\left(p-\omega-\omega^{\prime}\right), \\
= & -e^{2} \pi \int_{0}^{\Lambda} \frac{k^{2} d k}{2 \pi^{2}} \int_{0}^{\pi} \frac{1}{2} \sin \theta d \theta \int_{0}^{2 \pi} \frac{d \phi}{2 \pi} \int_{-k}^{k} d \omega\left\{\left(\beta_{+}(\omega)+\beta_{-}(\omega)\right)-\frac{1}{q}\left(\hat{k} \cdot q-2 \hat{k}_{1} q_{1}\right)\left(\beta_{+}(\omega)-\beta_{-}(\omega)\right)\right\} \\
& \times \delta(p-\omega-q), \\
\left.\operatorname{Im} \Pi_{0}^{22}\right|_{p o l e-c u t}= & 2 e^{2} \pi\left(1-e^{p / T}\right) \int \frac{d^{3} k}{(2 \pi)^{3}} \int_{-\infty}^{\infty} \int_{-\infty}^{\infty} d \omega d \omega^{\prime} n_{F}(\omega) n_{F}\left(\omega^{\prime}\right)\left\{\delta\left(\omega^{\prime}-q\right) \Theta\left(k^{2}-\omega^{2}\right)\left(\beta_{+}(\omega)+\beta_{-}(\omega)\right)\right. \\
& \left.-\frac{1}{q}\left(\hat{k} \cdot q-2 \hat{k}_{2} q_{2}\right) \delta\left(\omega^{\prime}-q\right) \Theta\left(k^{2}-\omega^{2}\right)\left(\beta_{+}(\omega)-\beta_{-}(\omega)\right)\right\} \delta\left(p-\omega-\omega^{\prime}\right) \\
= & -e^{2} \pi \int_{0}^{\Lambda} \frac{k^{2} d k}{2 \pi^{2}} \int_{0}^{\pi} \frac{1}{2} \sin \theta d \theta \int_{0}^{2 \pi} \frac{d \phi}{2 \pi} \int_{-k}^{k} d \omega\left\{\left(\beta_{+}(\omega)+\beta_{-}(\omega)\right)-\frac{1}{q}\left(\hat{k}_{k} \cdot q-2 \hat{k}_{2} q_{2}\right)\left(\beta_{+}(\omega)-\beta_{-}(\omega)\right)\right\} \\
& \times \delta(p-\omega-q),
\end{aligned}
$$




$$
\begin{aligned}
\left.\operatorname{Im} \Pi_{0}^{33}\right|_{\text {pole }- \text { cut }}= & 2 e^{2} \pi\left(1-e^{p / T}\right) \int \frac{d^{3} k}{(2 \pi)^{3}} \int_{-\infty}^{\infty} \int_{-\infty}^{\infty} d \omega d \omega^{\prime} n_{F}(\omega) n_{F}\left(\omega^{\prime}\right)\left\{\delta\left(\omega^{\prime}-q\right) \Theta\left(k^{2}-\omega^{2}\right)\left(\beta_{+}(\omega)+\beta_{-}(\omega)\right)\right. \\
& \left.-\frac{1}{q}\left(\hat{k}_{\perp} q_{\perp}-\hat{k}_{3} q_{3}\right) \delta\left(\omega^{\prime}-q\right) \Theta\left(k^{2}-\omega^{2}\right)\left(\beta_{+}(\omega)-\beta_{-}(\omega)\right)\right\} \delta\left(p-\omega-\omega^{\prime}\right) \\
= & -e^{2} \pi \int_{0}^{\Lambda} \frac{k^{2} d k}{2 \pi^{2}} \int_{0}^{\pi} \frac{1}{2} \sin \theta d \theta \int_{0}^{2 \pi} \frac{d \phi}{2 \pi} \int_{-k}^{k} d \omega\left\{\left(\beta_{+}(\omega)+\beta_{-}(\omega)\right)-\frac{1}{q}\left(\hat{k}_{\perp} q_{\perp}-\hat{k}_{3} q_{3}\right)\left(\beta_{+}(\omega)-\beta_{-}(\omega)\right)\right\} \\
& \times \delta(p-\omega-q),
\end{aligned}
$$

$$
\begin{aligned}
\left.\operatorname{Im} \prod_{0}^{13}\right|_{\text {pole-cut }}= & 2 e^{2} \pi\left(1-e^{p / T}\right) \int \frac{d^{3} k}{(2 \pi)^{3}} \int_{-\infty}^{\infty} \int_{-\infty}^{\infty} d \omega d \omega^{\prime} n_{F}(\omega) n_{F}\left(\omega^{\prime}\right)\left\{\frac{\hat{k}_{1} q_{3}+q_{1} \hat{k}_{3}}{q} \delta\left(\omega^{\prime}-q\right)\right. \\
& \left.\times\left(\beta_{+}(\omega)-\beta_{-}(\omega)\right) \Theta\left(k^{2}-\omega^{2}\right)\right\} \delta\left(p-\omega-\omega^{\prime}\right) \\
= & -e^{2} \pi \int_{0}^{\Lambda} \frac{k^{2} d k}{2 \pi^{2}} \int_{0}^{\pi} \frac{1}{2} \sin \theta d \theta \int_{0}^{2 \pi} \frac{d \phi}{2 \pi} \int_{-k}^{k} d \omega \frac{1}{q}\left(\hat{k}_{1} q_{3}+q_{1} \hat{k}_{3}\right)\left(\beta_{+}(\omega)-\beta_{-}(\omega)\right) \delta(p-\omega-q) .
\end{aligned}
$$

Here we note that the terms with $\delta\left(\omega^{\prime}+q\right) \delta\left(p-\omega-\omega^{\prime}\right) \Theta\left(k^{2}-\omega^{2}\right)$ will not contribute because $k^{2}-(p+q)^{2}$ cannot be greater than zero. So we have excluded those terms.

\section{B. Imaginary part of magnetic field dependent part of $\mathcal{O}\left[(e B)^{2}\right]$}

Similar to $\mathcal{O}\left[(e B)^{0}\right]$ case, the imaginary part of $\Pi_{2}^{11}, \Pi_{2}^{22}, \Pi_{2}^{33}$ and $\Pi_{2}^{13}$ can be written as

$$
\begin{aligned}
& \operatorname{Im} \Pi_{2}^{11}=-8 e^{2} \pi\left(1-e^{p / T}\right) \int \frac{d^{3} k}{(2 \pi)^{3}} \int_{-\infty}^{\infty} \int_{-\infty}^{\infty} d \omega d \omega^{\prime} n_{F}(\omega) n_{F}\left(\omega^{\prime}\right) \\
& \times\left[e B\left\{q_{3} \rho_{1}^{(0)}\left(\rho_{9}^{(1)}+\rho_{10}-\rho_{7}\right)-\rho_{1}^{(1)}\left(\rho_{8}+k_{3} \rho_{9}^{(0)}\right)\right\}+(e B)^{2}\left(\hat{k}_{1} q_{1}-\hat{k}_{2} q_{2}\right) \rho_{2}^{(0)}\left(\rho_{D_{-}}-\rho_{D_{+}}\right)\right. \\
& -(e B)^{2} q_{\perp}^{2}\left\{\rho_{3}^{(1)}\left(\rho_{D_{+}}+\rho_{D_{-}}\right)+\left(2 \hat{k}_{1} q_{1}-\hat{k} \cdot q\right) \rho_{3}^{(0)}\left(\rho_{D_{+}}-\rho_{D_{-}}\right)\right\} \\
& \left.+\rho_{0}^{(1)}\left(\rho_{15}^{(1)}-\rho_{14}^{(1)}+\rho_{16}-\rho_{13}-\rho_{11}\right)+\left(2 k_{1} q_{1}-\vec{k} \cdot \vec{q}\right) \rho_{0}^{(0)}\left(\rho_{15}^{(0)}-\rho_{14}^{(0)}\right)-q_{3} \rho_{0}^{(0)} \rho_{12}\right] \delta\left(p-\omega-\omega^{\prime}\right), \\
& \operatorname{Im} \Pi_{2}^{22}=-8 e^{2} \pi\left(1-e^{p / T}\right) \int \frac{d^{3} k}{(2 \pi)^{3}} \int_{-\infty}^{\infty} \int_{-\infty}^{\infty} d \omega d \omega^{\prime} n_{F}(\omega) n_{F}\left(\omega^{\prime}\right) \\
& \times\left[e B\left\{q_{3} \rho_{1}^{(0)}\left(\rho_{9}^{(1)}+\rho_{10}-\rho_{7}\right)-\rho_{1}^{(1)}\left(\rho_{8}+k_{3} \rho_{9}^{(0)}\right)\right\}+(e B)^{2}\left(\hat{k}_{2} q_{2}-\hat{k}_{1} q_{1}\right) \rho_{2}^{(0)}\left(\rho_{D_{-}}-\rho_{D_{+}}\right)\right. \\
& -(e B)^{2} q_{\perp}^{2}\left\{\rho_{3}^{(1)}\left(\rho_{D_{+}}+\rho_{D_{-}}\right)+\left(2 \hat{k}_{2} q_{2}-\hat{k} \cdot q\right) \rho_{3}^{(0)}\left(\rho_{D_{+}}-\rho_{D_{-}}\right)\right\}+\rho_{0}^{(1)}\left(\rho_{15}^{(1)}-\rho_{14}^{(1)}+\rho_{16}-\rho_{13}-\rho_{11}\right) \\
& \left.+\left(2 k_{2} q_{2}-\vec{k} \cdot \vec{q}\right) \rho_{0}^{(0)}\left(\rho_{15}^{(0)}-\rho_{14}^{(0)}\right)-q_{3} \rho_{0}^{(0)} \rho_{12}\right] \delta\left(p-\omega-\omega^{\prime}\right) \text {, } \\
& \operatorname{Im} \Pi_{2}^{33}=-8 e^{2} \pi\left(1-e^{p / T}\right) \int \frac{d^{3} k}{(2 \pi)^{3}} \int_{-\infty}^{\infty} \int_{-\infty}^{\infty} d \omega d \omega^{\prime} n_{F}(\omega) n_{F}\left(\omega^{\prime}\right) \\
& \times\left[e B\left\{q_{3} \rho_{1}^{(0)}\left(\rho_{9}^{(1)}+\rho_{10}-\rho_{7}\right)+\rho_{1}^{(1)}\left(\rho_{8}+k_{3} \rho_{9}^{(0)}\right)\right\}+(e B)^{2}\left(q_{3} \hat{k}_{3}-\hat{k} \cdot q\right) \rho_{2}^{(0)}\left(\rho_{D_{-}}-\rho_{D_{+}}\right)\right. \\
& -(e B)^{2} q_{\perp}^{2}\left\{\rho_{3}^{(1)}\left(\rho_{D_{+}}+\rho_{D_{-}}\right)+\left(2 q_{3} \hat{k}_{3}-\hat{k} \cdot q\right) \rho_{3}^{(0)}\left(\rho_{D_{+}}-\rho_{D_{-}}\right)\right\} \\
& \left.+\rho_{0}^{(1)}\left(\rho_{15}^{(1)}-\rho_{14}^{(1)}+\rho_{16}-\rho_{13}-\rho_{11}\right)+\left(2 k_{3} q_{3}-\vec{k} \cdot \vec{q}\right) \rho_{0}^{(0)}\left(\rho_{15}^{(0)}-\rho_{14}^{(0)}\right)+q_{3} \rho_{0}^{(0)} \rho_{12}\right] \delta\left(p-\omega-\omega^{\prime}\right) \text {, } \\
& \operatorname{Im} \Pi_{2}^{13}=-8 e^{2} \pi\left(1-e^{p / T}\right) \int \frac{d^{3} k}{(2 \pi)^{3}} \int_{-\infty}^{\infty} \int_{-\infty}^{\infty} d \omega d \omega^{\prime} n_{F}(\omega) n_{F}\left(\omega^{\prime}\right) \\
& \times\left[e B\left\{\rho_{1}^{(1)} k_{3} \rho_{9}^{(0)}\right\}+(e B)^{2} \hat{k}_{3} q_{1} \rho_{2}^{(0)}\left\{\rho_{D_{-}}-\rho_{D_{+}}\right\}-(e B)^{2} q_{\perp}^{2}\left\{\left(\hat{k}_{1} q_{3}+\hat{k}_{3} q_{1}\right) \rho_{3}^{(0)}\left(\rho_{D_{+}}-\rho_{D_{-}}\right)\right\}\right. \\
& \left.+\left(k_{1} q_{3}+k_{3} q_{1}\right) \rho_{0}^{(0)}\left(\rho_{15}^{(0)}-\rho_{14}^{(0)}\right)+q_{1} \rho_{0}^{(0)} \rho_{12}\right] \delta\left(p-\omega-\omega^{\prime}\right) \text {. }
\end{aligned}
$$


Various spectral functions are obtained in Appendix. As discussed before we also note that the imaginary part of various components of the self-energy contain the pole-pole and the pole-cut contributions. As explained earlier the phase space does not allow the pole-pole part to contribute in this order. In $\mathcal{O}\left[(e B)^{2}\right]$ the contribution comes only from the pole-cut part.

\section{Pole-cut part of $\mathcal{O}\left[(e B)^{2}\right]$}

Now the expressions of pole-cut parts of Eqs. (63), (64), (65), and (66) after using the approximations, are given below:

$$
\begin{aligned}
\left.\operatorname{Im} \Pi_{2}^{11}\right|_{\text {pole }- \text { cut }}= & 4 e^{2} \pi \int_{0}^{\Lambda} \frac{k^{2} d k}{2 \pi^{2}} \int \frac{1}{2} \sin \theta d \theta \int \frac{d \phi}{2 \pi} \int_{-k}^{k} d \omega \int_{-\infty}^{\infty} d \omega^{\prime}\left[\delta ^ { \prime \prime \prime } ( \omega ^ { \prime } - \omega _ { q } ) \left\{-\frac{(e B)^{2} q_{\perp}^{2}}{96 \omega_{q}^{3}}\left(\beta_{+}+\beta_{-}\right)\right.\right. \\
& \left.-\frac{(e B)^{2} q_{\perp}^{2}\left(2 \hat{k}_{1} q_{1}-\hat{k} \cdot q\right)}{96 \omega_{q}^{4}}\left(\beta_{+}-\beta_{-}\right)\right\} \\
& -\delta^{\prime \prime}\left(\omega^{\prime}-\omega_{q}\right)\left\{\frac{3(e B)^{2}}{64 \omega_{q}^{3}}\left(\frac{q_{\perp}^{2}\left(\hat{k}_{1} q_{1}-\hat{k} \cdot q\right)}{\omega_{q}^{2}}-\left(\hat{k}_{1} q_{1}-\hat{k}_{2} q_{2}\right)\right)\left(\beta_{+}-\beta_{-}\right)+\frac{3(e B)^{2} q_{\perp}^{2}}{128 \omega_{q}^{4}}\left(\beta_{+}+\beta_{-}\right)\right\} \\
& +\delta^{\prime}\left(\omega^{\prime}-\omega_{q}\right)\left\{\frac{e B q_{3}}{4 \omega_{q}^{2}}\left(\beta_{9}^{(1)}+\beta_{10}-\beta_{7}\right)-\frac{e B}{4 \omega_{q}}\left(\beta_{8}+k_{3} \beta_{9}^{(0)}\right)-\frac{(e B)^{2}}{16 \omega_{q}^{4}}\left(\hat{k}_{2} q_{2}-\hat{k}_{1} q_{1}+\frac{5 q_{\perp}^{2}\left(\hat{k}_{1} q_{1}-\hat{k} \cdot q\right)}{2 \omega_{q}^{2}}\right)\left(\beta_{+}-\beta_{-}\right)\right. \\
& \left.-\frac{(e B)^{2} q_{\perp}^{2}}{32 \omega_{q}^{5}}\left(\beta_{+}+\beta_{-}\right)\right\} \\
& +\delta\left(\omega^{\prime}-\omega_{q}\right)\left\{\frac{e B q_{3}}{4 \omega_{q}^{3}}\left(\beta_{9}^{(1)}+\beta_{10}-\beta_{7}\right)-\frac{(e B)^{2}}{16 \omega_{q}^{5}}\left(3 \hat{k}_{2} q_{2}-3 \hat{k}_{1} q_{1}+\frac{5 q_{\perp}^{2}\left(\hat{k}_{1} q_{1}-\hat{k} \cdot q\right)}{2 \omega_{q}^{2}}\right)\left(\beta_{+}-\beta_{-}\right)\right. \\
& \left.\left.-\frac{1}{2}\left(\beta_{15}^{(1)}+\beta_{16}-\left(\beta_{14}^{(1)}+\beta_{11}+\beta_{13}\right)\right)-\frac{\left(2 k_{1} q_{1}-\vec{k} \cdot \vec{q}\right)}{2 \omega_{q}}\left(\beta_{15}^{(0)}-\beta_{14}^{(0)}\right)+\frac{q_{3}}{2 \omega_{q}} \beta_{12}\right\}\right] \delta\left(p-\omega-\omega^{\prime}\right)
\end{aligned}
$$

$$
\begin{aligned}
\left.\operatorname{Im} \Pi_{2}^{22}\right|_{\text {pole }- \text { cut }}= & 4 e^{2} \pi \int_{0}^{\Lambda} \frac{k^{2} d k}{2 \pi^{2}} \int \frac{1}{2} \sin \theta d \theta \int \frac{d \phi}{2 \pi} \int_{-k}^{k} d \omega \int_{-\infty}^{\infty} d \omega^{\prime}\left[\delta ^ { \prime \prime \prime } ( \omega ^ { \prime } - \omega _ { q } ) \left\{-\frac{(e B)^{2} q_{\perp}^{2}}{96 \omega_{q}^{3}}\left(\beta_{+}+\beta_{-}\right)\right.\right. \\
& \left.-\frac{(e B)^{2} q_{\perp}^{2}\left(2 \hat{k}_{2} q_{2}-\hat{k} \cdot q\right)}{96 \omega_{q}^{4}}\left(\beta_{+}-\beta_{-}\right)\right\} \\
& -\delta^{\prime \prime}\left(\omega^{\prime}-\omega_{q}\right)\left\{\frac{3(e B)^{2}}{64 \omega_{q}^{3}}\left(\frac{q_{\perp}^{2}\left(\hat{k}_{2} q_{2}-\hat{k} \cdot q\right)}{\omega_{q}^{2}}-\left(\hat{k}_{2} q_{2}-\hat{k}_{1} q_{1}\right)\right) \times\left(\beta_{+}-\beta_{-}\right)+\frac{3(e B)^{2} q_{\perp}^{2}}{128 \omega_{q}^{4}}\left(\beta_{+}+\beta_{-}\right)\right\} \\
& +\delta^{\prime}\left(\omega^{\prime}-\omega_{q}\right)\left\{\frac{e B q_{3}}{4 \omega_{q}^{2}}\left(\beta_{9}^{(1)}+\beta_{10}-\beta_{7}\right)-\frac{e B}{4 \omega_{q}}\left(\beta_{8}+k_{3} \beta_{9}^{(0)}\right)-\frac{(e B)^{2}}{16 \omega_{q}^{4}}\left(\hat{k}_{1} q_{1}-\hat{k}_{2} q_{2}+\frac{5 q_{\perp}^{2}\left(\hat{k}_{2} q_{2}-\hat{k} \cdot q\right)}{2 \omega_{q}^{2}}\right)\left(\beta_{+}-\beta_{-}\right)\right. \\
& \left.-\frac{(e B)^{2} q_{\perp}^{2}}{32 \omega_{q}^{5}}\left(\beta_{+}+\beta_{-}\right)\right\} \\
& +\delta\left(\omega^{\prime}-\omega_{q}\right)\left\{\frac{e B q_{3}}{4 \omega_{q}^{3}}\left(\beta_{9}^{(1)}+\beta_{10}-\beta_{7}\right)-\frac{(e B)^{2}}{16 \omega_{q}^{5}}\left(3 \hat{k}_{1} q_{1}-3 \hat{k}_{2} q_{2}+\frac{5 q_{\perp}^{2}\left(\hat{k}_{2} q_{2}-\hat{k} \cdot q\right)}{2 \omega_{q}^{2}}\right)\left(\beta_{+}-\beta_{-}\right)\right. \\
& \left.\left.-\frac{1}{2}\left(\beta_{15}^{(1)}+\beta_{16}-\left(\beta_{14}^{(1)}+\beta_{11}+\beta_{13}\right)\right)-\frac{\left(2 k_{2} q_{2}-\vec{k} \cdot \vec{q}\right)}{2 \omega_{q}}\left(\beta_{15}^{(0)}-\beta_{14}^{(0)}\right)+\frac{q_{3}}{2 \omega_{q}} \beta_{12}\right\}\right] \delta\left(p-\omega-\omega^{\prime}\right)
\end{aligned}
$$




$$
\begin{aligned}
& \left.\operatorname{Im} \Pi_{2}^{33}\right|_{\text {pole-cut }}=4 e^{2} \pi \int_{0}^{\Lambda} \frac{k^{2} d k}{2 \pi^{2}} \int \frac{1}{2} \sin \theta d \theta \int \frac{d \phi}{2 \pi} \int_{-k}^{k} d \omega \int_{-\infty}^{\infty} d \omega^{\prime}\left[\delta ^ { \prime \prime \prime } ( \omega ^ { \prime } - \omega _ { q } ) \left\{-\frac{(e B)^{2} q_{\perp}^{2}}{96 \omega_{q}^{3}}\left(\beta_{+}+\beta_{-}\right)\right.\right. \\
& \left.-\frac{(e B)^{2} q_{\perp}^{2}\left(\hat{k}_{3} q_{3}-\hat{k}_{\perp} q_{\perp}\right)}{96 \omega_{q}^{4}}\left(\beta_{+}-\beta_{-}\right)\right\} \\
& -\delta^{\prime \prime}\left(\omega^{\prime}-\omega_{q}\right)\left\{\frac{3(e B)^{2}}{64 \omega_{q}^{3}}\left(\frac{q_{\perp}^{2}\left(\hat{k}_{3} q_{3}-\hat{k}_{\perp} q_{\perp}\right)}{\omega_{q}^{2}}+\hat{k}_{\perp} q_{\perp}\right) \times\left(\beta_{+}-\beta_{-}\right)+\frac{3(e B)^{2} q_{\perp}^{2}}{128 \omega_{q}^{4}}\left(\beta_{+}+\beta_{-}\right)\right\} \\
& +\delta^{\prime}\left(\omega^{\prime}-\omega_{q}\right)\left\{\frac { e B q _ { 3 } } { 4 \omega _ { q } ^ { 2 } } \left(\left(\beta_{9}^{(1)}+\beta_{10}-\beta_{7}\right)+\frac{e B}{4 \omega_{q}}\left(\beta_{8}+k_{3} \rho_{9}^{(0)}\right)\right.\right. \\
& \left.-\frac{(e B)^{2}}{16 \omega_{q}^{4}}\left(\hat{k}_{\perp} q_{\perp}+\frac{5 q_{\perp}^{2}\left(\hat{k}_{3} q_{3}-\hat{k}_{\perp} q_{\perp}\right)}{2 \omega_{q}^{2}}\right)\left(\beta_{+}-\beta_{-}\right)-\frac{(e B)^{2} q_{\perp}^{2}}{32 \omega_{q}^{5}}\left(\beta_{+}+\beta_{-}\right)\right\} \\
& +\delta\left(\omega^{\prime}-\omega_{q}\right)\left\{\frac{e B q_{3}}{4 \omega_{q}^{3}}\left(\beta_{9}^{(1)}+\beta_{10}-\beta_{7}\right)-\frac{(e B)^{2}}{16 \omega_{q}^{5}}\left(3 \hat{k}_{\perp} q_{\perp}+\frac{5 q_{\perp}^{2}\left(\hat{k}_{3} q_{3}-\hat{k}_{\perp} q_{\perp}\right)}{2 \omega_{q}^{2}}\right)\left(\beta_{+}-\beta_{-}\right)\right. \\
& \left.\left.-\frac{1}{2}\left(\beta_{15}^{(1)}+\beta_{16}-\left(\beta_{14}^{(1)}+\beta_{13}+\beta_{11}\right)\right)-\frac{\left(2 k_{3} q_{3}-\vec{k} \cdot \vec{q}\right)}{2 \omega_{q}}\left(\beta_{15}^{(0)}-\beta_{14}^{(0)}\right)-\frac{q_{3}}{2 \omega_{q}} \beta_{12}\right\}\right] \delta\left(p-\omega-\omega^{\prime}\right), \\
& \times\left\{-\frac{(e B)^{2} q_{\perp}^{2}\left(\hat{k}_{1} q_{3}+\hat{k}_{3} q_{1}\right)}{96 \omega_{q}^{4}}\left(\beta_{+}-\beta_{-}\right)\right\}-\delta^{\prime \prime}\left(\omega^{\prime}-\omega_{q}\right)\left\{\frac{3(e B)^{2}}{64 \omega_{q}^{3}}\left(\frac{q_{\perp}^{2}\left(\hat{k}_{1} q_{3}+\hat{k}_{3} q_{1}\right)}{\omega_{q}^{2}}-\hat{k}_{3} q_{1}\right)\left(\beta_{+}-\beta_{-}\right)\right\} \\
& +\delta^{\prime}\left(\omega^{\prime}-\omega_{q}\right)\left\{\frac{e B}{4 \omega_{q}} k_{1} \beta_{9}^{(0)}-\frac{(e B)^{2}}{16 \omega_{q}^{4}}\left(-\hat{k}_{3} q_{1}+\frac{5 q_{\perp}^{2}\left(\hat{k}_{1} q_{3}+\hat{k}_{3} q_{1}\right)}{2 \omega_{q}^{2}}\right)\left(\beta_{+}-\beta_{-}\right)\right\} \\
& +\delta\left(\omega^{\prime}-\omega_{q}\right)\left\{-\frac{(e B)^{2}}{16 \omega_{q}^{5}}\left(-3 \hat{k}_{3} q_{1}+\frac{5 q_{\perp}^{2}\left(\hat{k}_{1} q_{3}+\hat{k}_{3} q_{1}\right)}{2 \omega_{q}^{2}}\right)\left(\beta_{+}-\beta_{-}\right)\right. \\
& \left.\left.-\frac{\left(k_{1} q_{3}+k_{3} q_{1}\right)}{2 \omega_{q}}\left(\beta_{15}^{(0)}-\beta_{14}^{(0)}\right)-\frac{q_{1}}{2 \omega_{q}} \beta_{12}\right\}\right] \delta\left(p-\omega-\omega^{\prime}\right) \text {. }
\end{aligned}
$$

\section{RESULTS}

We perform the integrations in Eqs. (59), (60), (61), (62), (67), (68), (69), and (70) numerically. In this calculation we have taken $m_{\pi}=0.14 \mathrm{GeV}$. The results are shown for $\Lambda=$ $0.25 \mathrm{GeV}$ which satisfies $e T \ll \Lambda \ll T$.

The damping rate of photon in presence of magnetic field depends on the angle, $\theta_{p}$, between the momentum of photon and the magnetic field. Figure 3 shows the variation of the damping rate of a hard photon with the propagation angle. It increases with the increasing propagation angle. One can see that the two transverse modes of a hard photon are damped in a similar fashion. Since the magnetic field strength is very weak, this difference appears to be very small. We note that the magnetic correction is $\sim \mathcal{O}\left[(e B)^{2}\right]$ and switching the magnetic field from $z$ to $-z$ direction would not affect the result. These two orientations of the magnetic field correspond to the propagation angle of photon $\theta_{p}$ and $\pi-\theta_{p}$. These two situations are identical which correspond to the same damping rates of photon at $\theta_{p}$ and $\pi-\theta_{p}$.

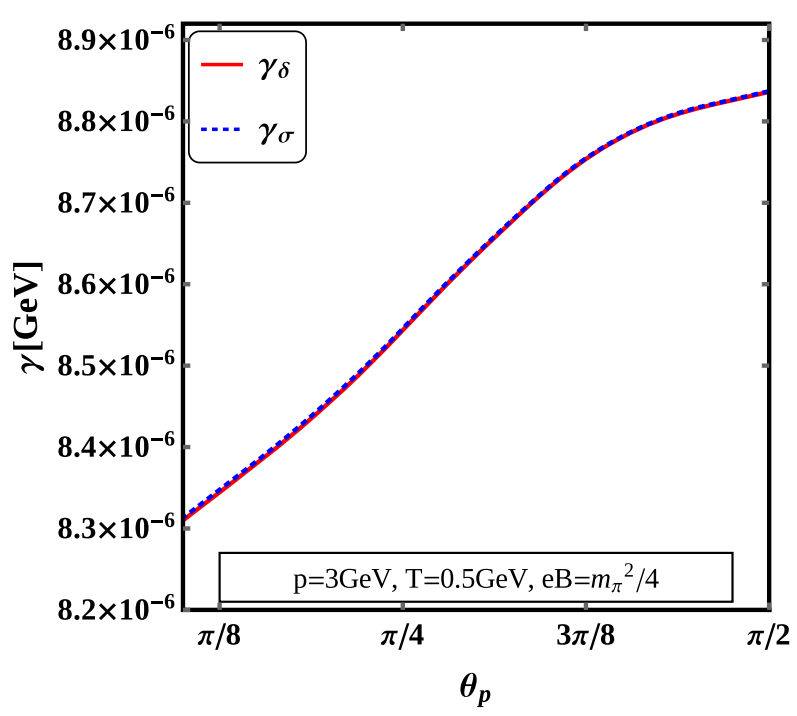

FIG. 3. Plot of damping rate of photon with the propagation angle $\theta_{p}$ for $p=3 \mathrm{GeV}, T=0.5 \mathrm{GeV}$ and $e B=m_{\pi}^{2} / 4$. 


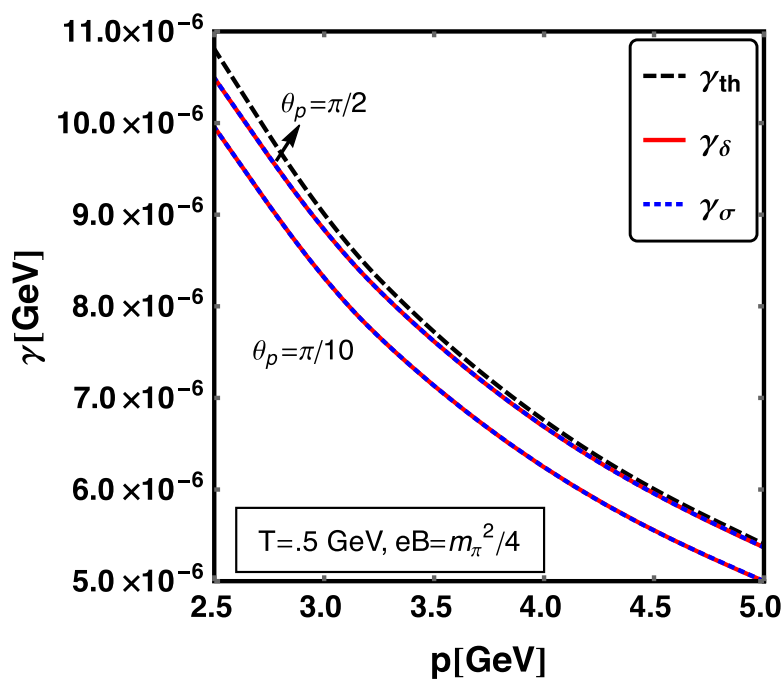

FIG. 4. Plot of damping rate of photon with the energy for $T=0.5 \mathrm{GeV}$ and $e B=m_{\pi}^{2} / 4$ at propagation angles $\theta_{p}=\pi / 10$ and $\pi / 2$.

In Fig. 4 we display the damping rate as a function of photon momentum for two propagation angles $\pi / 10$ and $\pi / 2$. The soft contribution of the damping rate in a thermal medium agrees well with that obtained in Ref. [45]. In presence of a thermomagnetic medium, the soft contribution to the damping rate is found to be reduced than that of the thermal one. For small propagation angle, the reduction of the damping rate is more compared to that of thermal medium. For higher momentum the damping rate approaches the thermal value as the temperature becomes the dominant scale as compared to the strength of the magnetic field considered.

Figure 5 displays the variation of damping rate with temperature for a specific value of momentum and magnetic

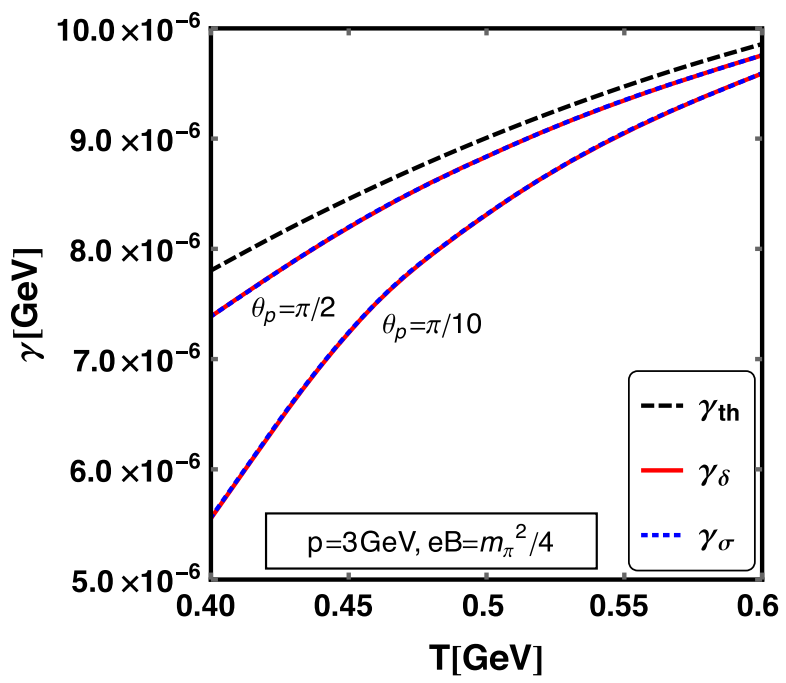

FIG. 5. Plot of damping rate of the hard photon with temperature at $p=3 \mathrm{GeV}$ and $e B=m_{\pi}^{2} / 4$ for two propagation angles $\pi / 10$ and $\pi / 2$.

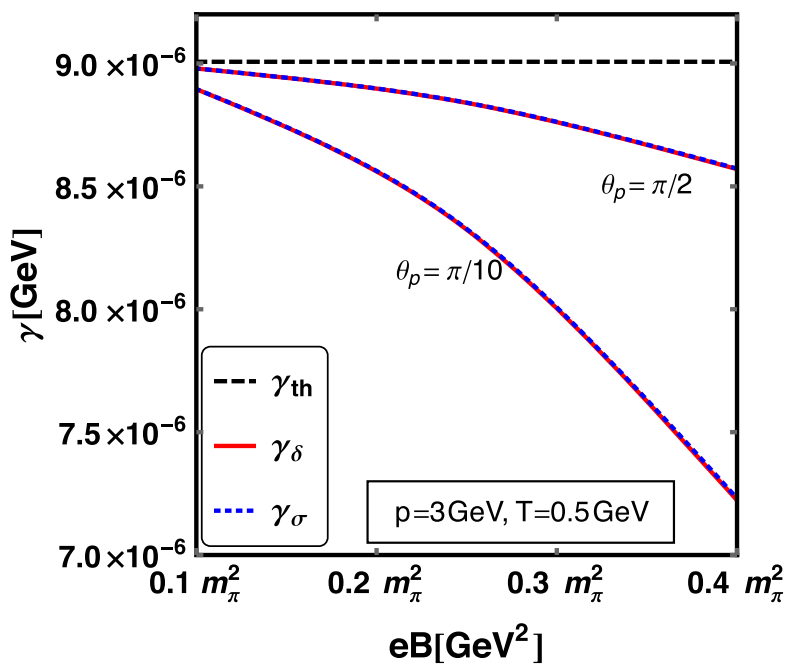

FIG. 6. Plot of damping rate of the hard photon with the magnetic field strength at $T=0.5 \mathrm{GeV}$ and $p=3 \mathrm{GeV}$ for two propagation angles $\pi / 10$ and $\pi / 2$.

field for two propagation angles $\pi / 10$ and $\pi / 2$. It is found that the soft contribution to the damping rate increases with the increase in temperature both in thermal and thermomagnetic medium. For small propagation angle the damping rate is more reduced compared to that of large propagation angle. This observation is consistent with Fig. 4.

Figure 6 shows the variation of the damping rate with the magnetic field strength for specific values of photon momentum and temperature for two propagation angles. The thermal damping rate $\left(\mathcal{O}\left[(e B)^{0}\right]\right)$ is represented by the black dashed horizontal line. The thermomagnetic damping rate decreases with the increasing magnetic field. At smaller propagation angles the photons are less damped

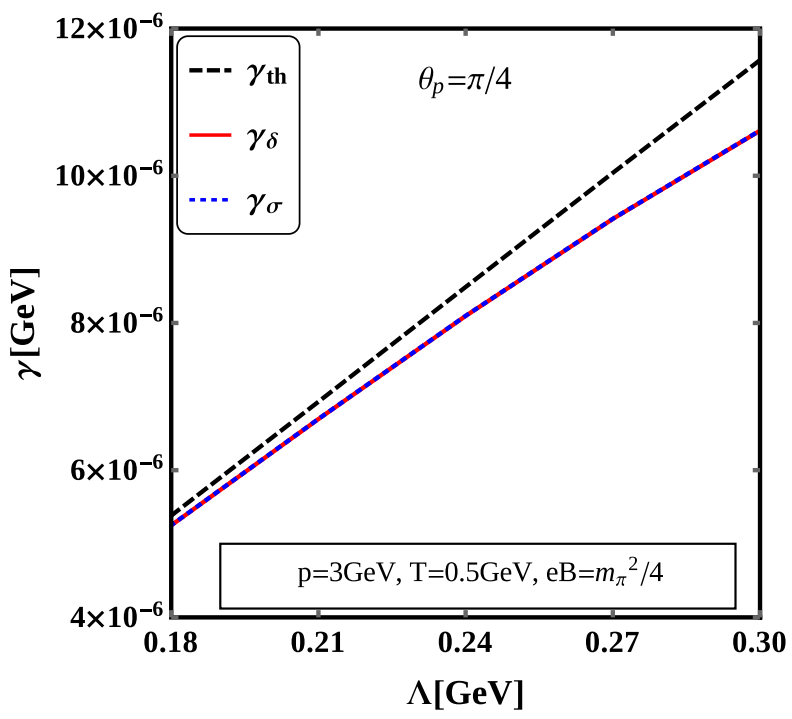

FIG. 7. Plot of damping rate of photon with $\Lambda$ for $\theta_{p}=\pi / 4$, $p=3 \mathrm{GeV}, T=0.5 \mathrm{GeV}$ and $e B=m_{\pi}^{2} / 4$. 
than that of higher propagation angles which are consistent with Fig. 4.

Figure 7 shows the variation of the photon damping rate with the separation scale $\Lambda$ keeping the scale hierarchy $e T \ll \Lambda \ll T$. As the allowed phase space increases with the increase of $\Lambda$, the damping rate is also found to increase with it. ${ }^{3}$ The magnetic correction to the thermal damping rate is negative. So, the difference between the thermal and thermomagnetic damping rate increases with $\Lambda$.

\section{CONCLUSION}

We have calculated the soft contribution to the damping rate of a hard photon in a weakly magnetized QED medium where momentum of one of the fermion in the loop is considered as soft. The two degenerate transverse modes of photon in thermal medium are damped in a similar fashion in presence of weak magnetic field as shown in Fig. 3. The difference between two transverse modes is very marginal due to weak field approximation. The soft contribution to the damping rate in the thermomagnetic medium is reduced compared to that of the thermal medium. When the magnetic field is switched off, the thermomagnetic damping modes reduce to its thermal value. The effect of magnetic field is found to be dominant at low temperature and low photon momentum.
The soft contribution to the hard photon damping rate is $\sim 10^{-6} \mathrm{GeV}$. Thus, a photon of a few $\mathrm{GeV}$ energy traversing in the QED medium of temperature $\sim 0.5 \mathrm{GeV}$ and background magnetic field $\sim 0.005 \mathrm{GeV}^{2}$ has a mean free path $\left(\lambda=\gamma^{-1} / 2\right)$ of a few $\AA$. When the present calculation is extended to the case of relativistic heavy ion collisions, the mean free path of photon is found to be of a few hundred $\mathrm{fm}$. This confirms that the mean free path of photon is larger than the size of the fireball and photon can be treated as a direct probe.

The damping rate is found to be dependent on the separation scale $\Lambda$. One needs to add the hard contribution with the soft contribution to cancel the $\Lambda$ dependence of the result. The hard contribution to the photon damping rate comes from two-loop order with hard particles in the loop having momentum of the order of or higher than the temperature. This itself is a huge calculation which is in progress.

\section{ACKNOWLEDGMENTS}

R. G. is funded by University Grants Commission (UGC). B. K. and M. G. M. were funded by Department of Atomic Energy (DAE), India via the project TPAES. R. G. and B. K. would like to thank Aritra Das for useful discussions.

\section{APPENDIX: SPECTRAL REPRESENTATION OF THE PROPAGATORS}

$$
\begin{aligned}
\lim _{\epsilon \rightarrow 0} \int_{-\infty}^{\infty} d x \frac{\epsilon}{x^{2}+\epsilon^{2}} f(x) & \approx f(0) \int_{-\infty}^{\infty} d x \frac{\epsilon}{x^{2}+\epsilon^{2}}\left\{\begin{array}{l}
\text { significant contribution comes from } \\
\text { integration, where } x \simeq 0
\end{array}\right. \\
& =f(0) \epsilon \int_{-\infty}^{\infty} d x \frac{1}{x^{2}+\epsilon^{2}}=\pi f(0),
\end{aligned}
$$

where $f(x)$ is a test function.

From the above equations we can write,

$$
\begin{aligned}
& \lim _{\epsilon \rightarrow 0} \frac{\epsilon}{x^{2}+\epsilon^{2}}=\pi \delta(x) \\
& \lim _{\epsilon \rightarrow 0} \operatorname{Im} \frac{1}{x+i \epsilon}=\frac{1}{2 i} \lim _{\epsilon \rightarrow 0}\left[\frac{1}{x+i \epsilon}-\frac{1}{x-i \epsilon}\right]=\frac{1}{2 i} \lim _{\epsilon \rightarrow 0} \frac{-2 i \epsilon}{x^{2}+\epsilon^{2}}=-\pi \delta(x) . \\
& \lim _{\epsilon \rightarrow 0} \int_{-\infty}^{\infty} d x \frac{2 \epsilon x}{\left(x^{2}+\epsilon^{2}\right)^{2}} f(x)=\lim _{\epsilon \rightarrow 0} \int_{-\infty}^{\infty} d x \epsilon f(x) \frac{d}{d x}\left[-\frac{1}{\left(x^{2}+\epsilon^{2}\right)}\right] \\
& =\lim _{\epsilon \rightarrow 0} \int_{-\infty}^{\infty} d x f^{\prime}(x) \frac{\epsilon}{x^{2}+\epsilon^{2}}=\pi f^{\prime}(0)=\pi \int d x f^{\prime}(x) \delta(x)=-\pi \int d x f(x) \delta^{\prime}(x) .
\end{aligned}
$$

From the above equation we find,

$$
\lim _{\epsilon \rightarrow 0} \frac{2 \epsilon x}{\left(x^{2}+\epsilon^{2}\right)^{2}}=-\pi \delta^{\prime}(x)
$$

\footnotetext{
${ }^{3}$ Nevertheless, the damping rate is expected to be $\Lambda$ independent when hard contribution is added.
} 
Now using Eq. (A5) one can calculate,

$$
\lim _{\epsilon \rightarrow 0} \operatorname{Im} \frac{1}{(x+i \epsilon)^{2}}=\frac{1}{2 i} \lim _{\epsilon \rightarrow 0}\left[\frac{1}{(x+i \epsilon)^{2}}-\frac{1}{(x-i \epsilon)^{2}}\right]=\frac{1}{2 i} \lim _{\epsilon \rightarrow 0} \frac{-i 4 \epsilon x}{\left(x^{2}+\epsilon^{2}\right)^{2}}=\pi \delta^{\prime}(x) .
$$

Similarly,

$$
\lim _{\epsilon \rightarrow 0} \int_{-\infty}^{\infty} d x \frac{\epsilon^{3}-3 x^{2} \epsilon}{\left(x^{2}+\epsilon^{2}\right)^{3}} f(x)=\lim _{\epsilon \rightarrow 0} \int_{-\infty}^{\infty} d x \frac{\epsilon^{3}}{\left(x^{2}+\epsilon^{2}\right)^{3}} f(x)-\lim _{\epsilon \rightarrow 0} \int_{-\infty}^{\infty} d x \frac{3 x^{2} \epsilon}{\left(x^{2}+\epsilon^{2}\right)^{3}} f(x)=I_{1}+I_{2},
$$

where

$$
\begin{aligned}
I_{1} & =\lim _{\epsilon \rightarrow 0} \int_{-\infty}^{\infty} d x \frac{\epsilon^{3} f(x)}{\left(x^{2}+\epsilon^{2}\right)^{3}} \approx \lim _{\epsilon \rightarrow 0} \epsilon^{3} f(0) \int_{-\infty}^{\infty} d x \frac{1}{\left(x^{2}+\epsilon^{2}\right)^{3}}=\lim _{\epsilon \rightarrow 0} \epsilon^{3} f(0) \frac{3}{8} \frac{1}{\epsilon^{5}}=\lim _{\epsilon \rightarrow 0} \frac{3}{8 \epsilon^{2}} f(0), \\
I_{2} & =-3 \lim _{\epsilon \rightarrow 0} \int_{-\infty}^{\infty} d x \frac{x^{2} \epsilon f(x)}{\left(x^{2}+\epsilon^{2}\right)^{3}}=-3 \lim _{\epsilon \rightarrow 0} \epsilon d x f(x)\left[\frac{1}{8} \frac{d^{2}}{d x^{2}}\left(\frac{1}{x^{2}+\epsilon^{2}}\right)+\frac{1}{8} \frac{2}{\left(x^{2}+\epsilon^{2}\right)^{2}}\right] \\
& =-\lim _{\epsilon \rightarrow 0} \frac{3}{8 \epsilon^{2}} f(0)-\frac{3}{8} \lim _{\epsilon \rightarrow 0} \epsilon \int d x f(x) \frac{d^{2}}{d x^{2}}\left(\frac{1}{x^{2}+\epsilon^{2}}\right) .
\end{aligned}
$$

So,

$$
\begin{aligned}
I_{1}+I_{2} & =-\frac{3}{8} \lim _{\epsilon \rightarrow 0} \epsilon \int d x f(x) \frac{d^{2}}{d x^{2}}\left(\frac{1}{x^{2}+\epsilon^{2}}\right)=\frac{3}{8} \lim _{\epsilon \rightarrow 0} \int d x \epsilon f^{\prime}(x) \frac{d}{d x}\left(\frac{1}{x^{2}+\epsilon^{2}}\right) \\
& =-\frac{3}{8} \lim _{\epsilon \rightarrow 0} \int d x \epsilon f^{\prime \prime}(x) \frac{1}{x^{2}+\epsilon^{2}}=-\frac{3}{8} \pi \int d x f^{\prime \prime}(x) \delta(x)=-\frac{3}{8} \pi \int_{-\infty}^{\infty} d x f(x) \delta^{\prime \prime}(x) .
\end{aligned}
$$

We can conclude from the last few steps that,

$$
\lim _{\epsilon \rightarrow 0} \frac{\epsilon^{3}-3 x^{2} \epsilon}{\left(x^{2}+\epsilon^{2}\right)^{3}}=-\frac{3}{8} \pi \delta^{\prime \prime}(x)
$$

Using Eq. (A10) we can find

$$
\lim _{\epsilon \rightarrow 0} \operatorname{Im} \frac{1}{(x+i \epsilon)^{3}}=\frac{1}{2 i} \lim _{\epsilon \rightarrow 0}\left[\frac{1}{(x+i \epsilon)^{2}}-\frac{1}{(x-i \epsilon)^{3}}\right]=\lim _{\epsilon \rightarrow 0} \frac{\epsilon^{3}-6 \epsilon x^{2}}{\left(x^{2}+\epsilon^{2}\right)^{3}}=-\frac{3}{8} \pi \delta^{\prime \prime}(x)
$$

Now,

$$
\begin{aligned}
\lim _{\epsilon \rightarrow 0} \operatorname{Im} \frac{1}{(x+i \epsilon)^{4}} & =\frac{1}{2 i} \lim _{\epsilon \rightarrow 0}\left[\frac{1}{(x+i \epsilon)^{4}}-\frac{1}{(x-i \epsilon)^{4}}\right]=\lim _{\epsilon \rightarrow 0} \frac{4 x \epsilon^{3}-4 x^{3} \epsilon}{\left(x^{2}+\epsilon^{2}\right)^{4}} \\
\lim _{\epsilon \rightarrow 0} \int_{-\infty}^{\infty} d x f(x) \frac{4 x \epsilon^{3}-4 x^{3} \epsilon}{\left(x^{2}+\epsilon^{2}\right)^{4}} & =\lim _{\epsilon \rightarrow 0} 4 \epsilon \int_{-\infty}^{\infty} d x f(x) \frac{1}{24} \frac{d^{3}}{d x^{3}}\left(\frac{1}{x^{2}+\epsilon^{2}}\right) \\
= & -\lim _{\epsilon \rightarrow 0} \frac{\epsilon}{6} \int_{-\infty}^{\infty} d x f^{\prime \prime \prime}(x) \frac{1}{x^{2}+\epsilon^{2}}=-\frac{\pi f^{\prime \prime \prime}(0)}{6}=\frac{\pi}{6} \int_{-\infty}^{\infty} d x f(x) \delta^{\prime \prime \prime}(x) . \\
& \text { Thus, } \lim _{\epsilon \rightarrow 0} \operatorname{Im} \frac{1}{(x+i \epsilon)^{4}}=\frac{\pi}{6} \delta^{\prime \prime \prime}(x) .
\end{aligned}
$$

Now we write the spectral representations for the free propagators as 


$$
\begin{aligned}
& \rho_{0}^{(1)}\left(\omega^{\prime}, q\right)=\frac{1}{2 \pi} \lim _{\epsilon \rightarrow 0} \operatorname{Im}\left[\frac{1}{\omega^{\prime}+i \epsilon+\omega_{q}}+\frac{1}{\omega^{\prime}+i \epsilon-\omega_{q}}\right]=-\frac{1}{2}\left[\delta\left(\omega^{\prime}+\omega_{q}\right)+\delta\left(\omega^{\prime}-\omega_{q}\right)\right], \\
& \rho_{0}^{(0)}\left(\omega^{\prime}, q\right)=\frac{1}{2 \pi \omega_{q}} \lim _{\epsilon \rightarrow 0} \operatorname{Im}\left[\frac{1}{\omega^{\prime}+i \epsilon-\omega_{q}}-\frac{1}{\omega^{\prime}+i \epsilon+\omega_{q}}\right]=\frac{1}{2 \omega_{q}}\left[\delta\left(\omega^{\prime}+\omega_{q}\right)-\delta\left(\omega^{\prime}-\omega_{q}\right)\right], \\
& \rho_{1}^{(1)}\left(\omega^{\prime}, q\right)=\frac{1}{4 \pi \omega_{q}} \lim _{\epsilon \rightarrow 0} \operatorname{Im}\left[\frac{1}{\left(\omega^{\prime}+i \epsilon-\omega_{q}\right)^{2}}-\frac{1}{\left(\omega^{\prime}+i \epsilon+\omega_{q}\right)^{2}}\right]=-\frac{1}{4 \omega_{q}}\left[\delta^{\prime}\left(\omega^{\prime}+\omega_{q}\right)-\delta^{\prime}\left(\omega^{\prime}-\omega_{q}\right)\right], \\
& \rho_{1}^{(0)}\left(\omega^{\prime}, q\right)=\frac{1}{4 \pi \omega_{q}^{2}} \lim _{\epsilon \rightarrow 0} \operatorname{Im}\left[\frac{1}{\left(\omega^{\prime}+i \epsilon+\omega_{q}\right)^{2}}+\frac{1}{\left(\omega^{\prime}+i \epsilon-\omega_{q}\right)^{2}}-\frac{1}{\omega_{q}}\left(\frac{1}{\omega^{\prime}+i \epsilon-\omega_{q}}-\frac{1}{\omega^{\prime}+i \epsilon+\omega_{q}}\right)\right] \\
& =\frac{1}{4 \omega_{q}^{2}}\left[\delta^{\prime}\left(\omega^{\prime}+\omega_{q}\right)+\delta^{\prime}\left(\omega^{\prime}-\omega_{q}\right)-\frac{1}{\omega_{q}}\left(\delta\left(\omega^{\prime}+\omega_{q}\right)-\delta\left(\omega^{\prime}-\omega_{q}\right)\right)\right] \text {, } \\
& \rho_{2}^{(1)}\left(\omega^{\prime}, q\right)=\frac{1}{8 \pi \omega_{q}^{2}} \lim _{\epsilon \rightarrow 0} \operatorname{Im}\left[\frac{1}{\left(\omega^{\prime}+i \epsilon+\omega_{q}\right)^{3}}+\frac{1}{\left(\omega^{\prime}+i \epsilon-\omega_{q}\right)^{3}}+\frac{1}{2 \omega_{q}}\left\{\frac{1}{\left(\omega^{\prime}+i \epsilon+\omega_{q}\right)^{2}}-\frac{1}{\left(\omega^{\prime}+i \epsilon-\omega_{q}\right)^{2}}\right\}\right] \\
& =\frac{1}{8 \omega_{q}^{2}}\left[-\frac{3}{8}\left(\delta^{\prime \prime}\left(\omega^{\prime}+\omega_{q}\right)+\delta^{\prime \prime}\left(\omega^{\prime}-\omega_{q}\right)\right)+\frac{1}{2 \omega_{q}}\left(\delta^{\prime}\left(\omega^{\prime}+\omega_{q}\right)-\delta^{\prime}\left(\omega^{\prime}-\omega_{q}\right)\right)\right] \text {, } \\
& \rho_{2}^{(0)}\left(\omega^{\prime}, q\right)=-\frac{1}{8 \pi \omega_{q}^{3}} \lim _{\epsilon \rightarrow 0} \operatorname{Im}\left[\frac{1}{\left(\omega^{\prime}+i \epsilon+\omega_{q}\right)^{3}}-\frac{1}{\left(\omega^{\prime}+i \epsilon-\omega_{q}\right)^{3}}\right. \\
& \left.+\frac{1}{2 \omega_{q}}\left\{\frac{1}{\left(\omega^{\prime}+i \epsilon+\omega_{q}\right)^{2}}+\frac{1}{\left(\omega^{\prime}+i \epsilon-\omega_{q}\right)^{2}}+\frac{3}{\omega_{q}}\left(\frac{1}{\omega^{\prime}+i \epsilon+\omega_{q}}-\frac{1}{\omega^{\prime}+i \epsilon-\omega_{q}}\right)\right\}\right] \\
& =-\frac{1}{8 \omega_{q}^{3}}\left[-\frac{3}{8}\left(\delta^{\prime \prime}\left(\omega^{\prime}+\omega_{q}\right)-\delta^{\prime \prime}\left(\omega^{\prime}-\omega_{q}\right)\right)+\frac{1}{2 \omega_{q}}\left\{\delta^{\prime}\left(\omega^{\prime}+\omega_{q}\right)+\delta^{\prime}\left(\omega^{\prime}-\omega_{q}\right)-\frac{3}{\omega_{q}}\left(\delta\left(\omega^{\prime}+\omega_{q}\right)-\delta\left(\omega^{\prime}-\omega_{q}\right)\right)\right\}\right] \text {, } \\
& \rho_{3}^{(1)}\left(\omega^{\prime}, q\right)=-\frac{1}{16 \pi \omega_{q}^{3}} \lim _{\epsilon \rightarrow 0} \operatorname{Im}\left[\frac{1}{\left(\omega^{\prime}+i \epsilon+\omega_{q}\right)^{4}}-\frac{1}{\left(\omega^{\prime}+i \epsilon-\omega_{q}\right)^{4}}\right. \\
& \left.+\frac{1}{\omega_{q}}\left\{\frac{1}{\left(\omega^{\prime}+i \epsilon+\omega_{q}\right)^{3}}+\frac{1}{\left(\omega^{\prime}+i \epsilon-\omega_{q}\right)^{3}}+\frac{1}{2 \omega_{q}}\left(\frac{1}{\left(\omega^{\prime}+i \epsilon+\omega_{q}\right)^{2}}-\frac{1}{\left(\omega^{\prime}+i \epsilon-\omega_{q}\right)^{2}}\right)\right\}\right] \\
& =-\frac{1}{16 \omega_{q}^{3}}\left[\frac{1}{6}\left(\delta^{\prime \prime \prime}\left(\omega^{\prime}+\omega_{q}\right)-\delta^{\prime \prime \prime}\left(\omega^{\prime}-\omega_{q}\right)\right)+\frac{1}{\omega_{q}}\left\{-\frac{3}{8}\left(\delta^{\prime \prime}\left(\omega^{\prime}+\omega_{q}\right)+\delta^{\prime \prime}\left(\omega^{\prime}-\omega_{q}\right)\right)+\frac{1}{2 \omega_{q}}\left(\delta^{\prime}\left(\omega^{\prime}+\omega_{q}\right)-\delta^{\prime}\left(\omega^{\prime}-\omega_{q}\right)\right)\right\},\right. \\
& \rho_{3}^{(0)}\left(\omega^{\prime}, q\right)=\frac{1}{16 \pi \omega_{q}^{4}} \lim _{\epsilon \rightarrow 0}\left[\frac{1}{\left(\omega^{\prime}+i \epsilon+\omega_{q}\right)^{4}}+\frac{1}{\left(\omega^{\prime}+i \epsilon-\omega_{q}\right)^{4}}+\frac{1}{2 \omega_{q}}\left\{\frac{4}{\left(\omega^{\prime}+i \epsilon+\omega_{q}\right)^{3}}-\frac{4}{\left(\omega^{\prime}+i \epsilon-\omega_{q}\right)^{3}}\right.\right. \\
& \left.\left.+\frac{1}{2 \omega_{q}}\left(\frac{10}{\left(\omega^{\prime}+i \epsilon+\omega_{q}\right)^{2}}+\frac{10}{\left(\omega^{\prime}+i \epsilon-\omega_{q}\right)^{2}}+\frac{1}{2 \omega_{q}}\left(\frac{20}{\omega^{\prime}+i \epsilon+\omega_{q}}-\frac{20}{\omega^{\prime}+i \epsilon-\omega_{q}}\right)\right)\right\}\right] \\
& =\frac{1}{16 \omega_{q}^{4}}\left[\frac{1}{6}\left(\delta^{\prime \prime \prime}\left(\omega^{\prime}+\omega_{q}\right)+\delta^{\prime \prime \prime}\left(\omega^{\prime}-\omega_{q}\right)\right)+\frac{1}{2 \omega_{q}}\left\{-\frac{3}{2}\left(\delta^{\prime \prime}\left(\omega^{\prime}+\omega_{q}\right)-\delta^{\prime \prime}\left(\omega^{\prime}-\omega_{q}\right)\right)+\frac{5}{\omega_{q}}\left(\delta^{\prime}\left(\omega^{\prime}+\omega_{q}\right)+\delta^{\prime}\left(\omega^{\prime}-\omega_{q}\right)\right.\right.\right. \\
& \left.\left.\left.-\frac{1}{\omega_{q}}\left(\delta\left(\omega^{\prime}+\omega_{q}\right)-\delta\left(\omega^{\prime}-\omega_{q}\right)\right)\right)\right\}\right] \text {. }
\end{aligned}
$$

The effective propagators are given as, 


$$
\begin{aligned}
& \frac{1}{D^{2}}=\frac{1}{D_{+} D_{-}}=\left.\sum_{i}\left(\frac{\partial D^{2}}{\partial \omega}\right)^{-1}\right|_{\omega=\omega_{i}} \frac{1}{\left(\omega-\omega_{i}\right)}, \\
& \frac{1}{D^{4}}=\frac{1}{\left(D_{+} D_{-}\right)^{2}}=\sum_{i}\left[\left.\left(\frac{\partial D^{2}}{\partial \omega}\right)^{-2}\right|_{\omega=\omega_{i}} \frac{1}{\left(\omega-\omega_{i}\right)^{2}}+\left.\frac{\partial}{\partial \omega}\left\{\frac{\left(\omega-\omega_{i}\right)^{2}}{D^{4}}\right\}\right|_{\omega=\omega_{i}} \frac{1}{\left(\omega-\omega_{i}\right)}\right] \\
& =\sum_{i}\left[\left.\left(\frac{\partial D^{2}}{\partial \omega}\right)^{-2}\right|_{\omega=\omega_{i}} \frac{1}{\left(\omega-\omega_{i}\right)^{2}}-\left.\frac{\partial^{2} D^{2}}{\partial \omega^{2}}\left(\frac{1}{3 !} \frac{\partial^{3} D^{6}}{\partial \omega^{3}}\right)^{-1}\right|_{\omega=\omega_{i}} \frac{1}{\left(\omega-\omega_{i}\right)}\right] \text {, } \\
& \frac{1}{D^{6}}=\frac{1}{\left(D_{+} D_{-}\right)^{3}}=\sum_{i}\left[\left.\left(\frac{\partial D^{2}}{\partial \omega}\right)^{-3}\right|_{\omega=\omega_{i}} \frac{1}{\left(\omega-\omega_{i}\right)^{3}}+\left.\frac{\partial}{\partial \omega}\left\{\frac{\left(\omega-\omega_{i}\right)^{3}}{D^{6}}\right\}\right|_{\omega=\omega_{i}} \frac{1}{\left(\omega-\omega_{i}\right)^{2}}+\left.\frac{1}{2} \frac{\partial^{2}}{\partial \omega^{2}}\left\{\frac{\left(\omega-\omega_{i}\right)^{3}}{D^{6}}\right\}\right|_{\omega=\omega_{i}} \frac{1}{\left(\omega-\omega_{i}\right)}\right] \\
& =\sum_{i}\left[\left.\left(\frac{\partial D^{2}}{\partial \omega}\right)^{-3}\right|_{\omega=\omega_{i}} \frac{1}{\left(\omega-\omega_{i}\right)^{3}}-\left.\frac{3}{2} \frac{\partial^{2} D^{2}}{\partial \omega^{2}}\left(\frac{1}{4 !} \frac{\partial^{4} D^{8}}{\partial \omega^{4}}\right)^{-1}\right|_{\omega=\omega_{i}} \frac{1}{\left(\omega-\omega_{i}\right)^{2}}\right. \\
& \left.-\left.\frac{3}{5}\left(\frac{\partial^{3} D^{2}}{\partial \omega^{3}}\left\{6\left(\frac{\partial^{4} D^{8}}{\partial \omega^{4}}\right)^{-1}+\frac{7}{12}\left(\frac{\partial D^{2}}{\partial \omega}\right)^{-4}\right\}+6 \frac{\partial^{2} D^{2}}{\partial \omega^{2}} \frac{\partial}{\partial \omega}\left(\frac{\partial^{4} D^{8}}{\partial \omega^{4}}\right)^{-1}\right)\right|_{\omega=\omega_{i}} \frac{1}{\left(\omega-\omega_{i}\right)}\right]
\end{aligned}
$$

where $\omega_{i}= \pm \omega_{ \pm}$are the poles of $D_{+}$and $D_{-}$.

The spectral functions of the dressed propagators are given as

$$
\rho_{D_{ \pm}}=-\frac{\left(\omega^{2}-k^{2}\right)}{2 m_{\mathrm{th}}^{2}}\left[\delta\left(\omega-\omega_{ \pm}\right)+\delta\left(\omega+\omega_{\mp}\right)\right]+\beta_{ \pm} \Theta\left(k^{2}-\omega^{2}\right),
$$

where

$$
\beta_{ \pm}=\frac{-\frac{1}{2}(k \mp \omega) m_{\mathrm{th}}^{2}}{\left[k(\omega \mp k)-m_{\mathrm{th}}^{2}\left\{Q_{0}\left(\frac{\omega}{k}\right) \mp Q_{1}\left(\frac{\omega}{k}\right)\right\}\right]^{2}+\left[\frac{1}{2}\left(1 \mp \frac{\omega}{k}\right) m_{\mathrm{th}}^{2} \pi\right]^{2}},
$$

where we use the Legendre function of second kind

$$
\begin{aligned}
& Q_{0}\left(\frac{\omega}{k}\right)=\frac{1}{2} \ln \left|\frac{\omega+k}{\omega-k}\right|-i \frac{\pi}{2} \Theta\left(k^{2}-\omega^{2}\right) . \\
& \rho_{4}(\omega, k)= \frac{1}{\pi} \operatorname{Im}\left(\frac{1}{D^{2}}\right)=-\left.\sum_{i}\left(\frac{\partial D^{2}}{\partial \omega}\right)^{-1}\right|_{\omega=\omega_{i}} \delta\left(\omega-\omega_{i}\right)+\beta_{4} \Theta\left(k^{2}-\omega^{2}\right) \\
&= \frac{\omega^{2}-k^{2}}{4 m_{\mathrm{th}}^{2}\left(k^{2}-\omega^{2}+m_{\mathrm{th}}^{2}\right)}\left[(\omega-k)\left(\delta\left(\omega-\omega_{+}\right)+\delta\left(\omega+\omega_{-}\right)\right)+(\omega+k)\left(\delta\left(\omega-\omega_{-}\right)+\delta\left(\omega+\omega_{+}\right)\right)\right]+\beta_{4} \Theta\left(k^{2}-\omega^{2}\right), \\
& \rho_{5}(\omega, k)= \frac{1}{\pi} \operatorname{Im}\left(\frac{1}{D^{4}}\right)=\sum_{i}\left[\left.\left(\frac{\partial D^{2}}{\partial \omega}\right)^{-2}\right|_{\omega=\omega_{i}} \delta^{\prime}\left(\omega-\omega_{i}\right)+\left.\frac{\partial^{2} D^{2}}{\partial \omega^{2}}\left(\frac{1}{3 !} \frac{\partial^{3} D^{6}}{\partial \omega^{3}}\right)^{-1}\right|_{\omega=\omega_{i}} \delta\left(\omega-\omega_{i}\right)\right]+\beta_{5} \Theta\left(k^{2}-\omega^{2}\right), \\
& \rho_{6}(\omega, k)= \frac{1}{\pi} \operatorname{Im}\left(\frac{1}{D^{6}}\right)=\sum_{i}\left[-\left.\frac{3}{8}\left(\frac{\partial D^{2}}{\partial \omega}\right)^{-3}\right|_{\omega=\omega_{i}} \delta^{\prime \prime}\left(\omega-\omega_{i}\right)-\left.\frac{3 \partial^{2} D^{2}}{\partial \omega^{2}}\left(\frac{1}{4 !} \frac{\partial^{4} D^{8}}{\partial \omega^{4}}\right)^{-1}\right|_{\omega=\omega_{i}} \delta^{\prime}\left(\omega-\omega_{i}\right)\right. \\
&\left.+\left.\frac{3}{5}\left(\frac{\partial^{3} D^{2}}{\partial \omega^{3}}\left\{6\left(\frac{\partial^{4} D^{8}}{\partial \omega^{4}}\right)^{-1}+\frac{7}{12}\left(\frac{\partial D^{2}}{\partial \omega}\right)^{-4}\right\}+6 \frac{\partial^{2} D^{2}}{\partial \omega^{2}} \frac{\partial}{\partial \omega}\left(\frac{\partial^{4} D^{8}}{\partial \omega^{4}}\right)^{-1}\right)\right|_{\omega=\omega_{i}} \delta\left(\omega-\omega_{i}\right)\right]+\beta_{6} \Theta\left(k^{2}-\omega^{2}\right), \\
& \rho_{7}(\omega, k)=\frac{1}{\pi} \operatorname{Im}\left(\frac{b^{\prime}}{D^{2}}\right)=-b^{\prime} \sum_{i}\left(\left.\frac{\partial D^{2}}{\partial \omega}\right|_{\omega=\omega_{i}}\right)^{-1} \delta\left(\omega-\omega_{i}\right)+\beta_{7} \Theta\left(k^{2}-\omega^{2}\right),
\end{aligned}
$$




$$
\begin{aligned}
& \rho_{8}(\omega, k)=\frac{1}{\pi} \operatorname{Im}\left(\frac{c^{\prime}}{D^{2}}\right)=-c^{\prime} \sum_{i}\left(\left.\frac{\partial D^{2}}{\partial \omega}\right|_{\omega=\omega_{i}}\right)^{-1} \delta\left(\omega-\omega_{i}\right)+\beta_{8} \Theta\left(k^{2}-\omega^{2}\right) \\
& \rho_{9}^{(0)}(\omega, k)=\frac{1}{\pi} \operatorname{Im}\left(\frac{h(1+a)}{D^{4}}\right)=h(1+a) \times \sum_{i}\left[\left(\left.\frac{\partial D^{2}}{\partial \omega}\right|_{\omega=\omega_{i}}\right)^{-2} \delta^{\prime}\left(\omega-\omega_{i}\right)-\left.\frac{\partial}{\partial \omega}\left\{\frac{\left(\omega-\omega_{i}\right)^{2}}{D^{4}}\right\}\right|_{\omega=\omega_{i}} \delta\left(\omega-\omega_{i}\right)\right] \\
& +\beta_{9}^{(0)} \Theta\left(k^{2}-\omega^{2}\right), \\
& \rho_{9}^{(1)}(\omega, k)=\frac{1}{\pi} \operatorname{Im}\left(\frac{h(1+a) \omega}{D^{4}}\right)=\omega h(1+a) \times \sum_{i}\left[\left(\left.\frac{\partial D^{2}}{\partial \omega}\right|_{\omega=\omega_{i}}\right)^{-2} \delta^{\prime}\left(\omega-\omega_{i}\right)-\left.\frac{\partial}{\partial \omega}\left\{\frac{\left(\omega-\omega_{i}\right)^{2}}{D^{4}}\right\}\right|_{\omega=\omega_{i}} \delta\left(\omega-\omega_{i}\right)\right] \\
& +\beta_{9}^{(1)} \Theta\left(k^{2}-\omega^{2}\right) \text {, } \\
& \rho_{10}(\omega, k)=\frac{1}{\pi} \operatorname{Im}\left(\frac{h b}{D^{4}}\right)=h b \times \sum_{i}\left[\left(\left.\frac{\partial D^{2}}{\partial \omega}\right|_{\omega=\omega_{i}}\right)^{-2} \delta^{\prime}\left(\omega-\omega_{i}\right)-\left.\frac{\partial}{\partial \omega}\left\{\frac{\left(\omega-\omega_{i}\right)^{2}}{D^{4}}\right\}\right|_{\omega=\omega_{i}} \delta\left(\omega-\omega_{i}\right)\right]+\beta_{10} \Theta\left(k^{2}-\omega^{2}\right) \\
& \rho_{11}(\omega, k)=\frac{1}{\pi} \operatorname{Im}\left[\frac{h b^{\prime}}{D^{4}}\right]=h b^{\prime} \times \sum_{i}\left[\left(\left.\frac{\partial D^{2}}{\partial \omega}\right|_{\omega=\omega_{i}}\right)^{-2} \delta^{\prime}\left(\omega-\omega_{i}\right)-\left.\frac{\partial}{\partial \omega}\left\{\frac{\left(\omega-\omega_{i}\right)^{2}}{D^{4}}\right\}\right|_{\omega=\omega_{i}} \delta\left(\omega-\omega_{i}\right)\right]+\beta_{11} \Theta\left(k^{2}-\omega^{2}\right) \\
& \rho_{12}(\omega, k)=\frac{1}{\pi} \operatorname{Im}\left[\frac{h c^{\prime}}{D^{4}}\right]=h c^{\prime} \times \sum_{i}\left[\left(\left.\frac{\partial D^{2}}{\partial \omega}\right|_{\omega=\omega_{i}}\right)^{-2} \delta^{\prime}\left(\omega-\omega_{i}\right)-\left.\frac{\partial}{\partial \omega}\left\{\frac{\left(\omega-\omega_{i}\right)^{2}}{D^{4}}\right\}\right|_{\omega=\omega_{i}} \delta\left(\omega-\omega_{i}\right)\right]+\beta_{12} \Theta\left(k^{2}-\omega^{2}\right), \\
& \rho_{13}(\omega, k)=\frac{1}{\pi} \operatorname{Im}\left[\frac{h^{\prime} b}{D^{4}}\right]=h^{\prime} b \times \sum_{i}\left[\left(\left.\frac{\partial D^{2}}{\partial \omega}\right|_{\omega=\omega_{i}}\right)^{-2} \delta^{\prime}\left(\omega-\omega_{i}\right)-\left.\frac{\partial}{\partial \omega}\left\{\frac{\left(\omega-\omega_{i}\right)^{2}}{D^{4}}\right\}\right|_{\omega=\omega_{i}} \delta\left(\omega-\omega_{i}\right)\right]+\beta_{13} \Theta\left(k^{2}-\omega^{2}\right), \\
& \rho_{14}^{(0)}(\omega, k)=\frac{1}{\pi} \operatorname{Im}\left[\frac{h^{\prime}(1+a)}{D^{4}}\right]=h^{\prime}(1+a) \sum_{i}\left[\left(\left.\frac{\partial D^{2}}{\partial \omega}\right|_{\omega=\omega_{i}}\right)^{-2} \delta^{\prime}\left(\omega-\omega_{i}\right)-\left.\frac{\partial}{\partial \omega}\left\{\frac{\left(\omega-\omega_{i}\right)^{2}}{D^{4}}\right\}\right|_{\omega=\omega_{i}} \delta\left(\omega-\omega_{i}\right)\right] \\
& +\beta_{14}^{(0)} \Theta\left(k^{2}-\omega^{2}\right), \\
& \rho_{14}^{(1)}(\omega, k)=\frac{1}{\pi} \operatorname{Im}\left[\frac{k_{0} h^{\prime}(1+a)}{D^{4}}\right]=\omega h^{\prime}(1+a) \sum_{i}\left[\left(\left.\frac{\partial D^{2}}{\partial \omega}\right|_{\omega=\omega_{i}}\right)^{-2} \delta^{\prime}\left(\omega-\omega_{i}\right)-\left.\frac{\partial}{\partial \omega}\left\{\frac{\left(\omega-\omega_{i}\right)^{2}}{D^{4}}\right\}\right|_{\omega=\omega_{i}} \delta\left(\omega-\omega_{i}\right)\right] \\
& +\beta_{14}^{(1)} \Theta\left(k^{2}-\omega^{2}\right), \\
& \rho_{15}^{(0)}(\omega, k)=\frac{1}{\pi} \operatorname{Im}\left[\frac{h^{2}(1+a)}{D^{6}}\right]=h^{2}(1+a) \times \sum_{i}\left[-\frac{3}{8}\left(\left.\frac{\partial D^{2}}{\partial \omega}\right|_{\omega=\omega_{i}}\right)^{-3} \delta^{\prime \prime}\left(\omega-\omega_{i}\right)+\left.\frac{\partial}{\partial \omega}\left\{\frac{\left(\omega-\omega_{i}\right)^{3}}{D^{6}}\right\}\right|_{\omega=\omega_{i}} \delta^{\prime}\left(\omega-\omega_{i}\right)\right. \\
& \left.-\left.\frac{\partial^{2}}{\partial \omega^{2}}\left\{\frac{\left(\omega-\omega_{i}\right)^{3}}{D^{6}}\right\}\right|_{\omega=\omega_{i}} \delta\left(\omega-\omega_{i}\right)\right]+\beta_{15}^{(0)} \Theta\left(k^{2}-\omega^{2}\right) \\
& \rho_{15}^{(1)}(\omega, k)=\frac{1}{\pi} \operatorname{Im}\left[\frac{h^{2}(1+a) k_{0}}{D^{6}}\right]=\omega h^{2}(1+a) \times \sum_{i}\left[-\frac{3}{8}\left(\left.\frac{\partial D^{2}}{\partial \omega}\right|_{\omega=\omega_{i}}\right)^{-3} \delta^{\prime \prime}\left(\omega-\omega_{i}\right)+\left.\frac{\partial}{\partial \omega}\left\{\frac{\left(\omega-\omega_{i}\right)^{3}}{D^{6}}\right\}\right|_{\omega=\omega_{i}} \delta^{\prime}\left(\omega-\omega_{i}\right)\right. \\
& \left.-\left.\frac{\partial^{2}}{\partial \omega^{2}}\left\{\frac{\left(\omega-\omega_{i}\right)^{3}}{D^{6}}\right\}\right|_{\omega=\omega_{i}} \delta\left(\omega-\omega_{i}\right)\right]+\beta_{15}^{(1)} \Theta\left(k^{2}-\omega^{2}\right) \\
& \rho_{16}(\omega, k)=\frac{1}{\pi} \operatorname{Im}\left[\frac{h^{2} b}{D^{6}}\right]=h^{2} b \times \sum_{i}\left[-\frac{3}{8}\left(\left.\frac{\partial D^{2}}{\partial \omega}\right|_{\omega=\omega_{i}}\right)^{-3} \delta^{\prime \prime}\left(\omega-\omega_{i}\right)+\left.\frac{\partial}{\partial \omega}\left\{\frac{\left(\omega-\omega_{i}\right)^{3}}{D^{6}}\right\}\right|_{\omega=\omega_{i}} \delta^{\prime}\left(\omega-\omega_{i}\right)\right. \\
& \left.-\left.\frac{\partial^{2}}{\partial \omega^{2}}\left\{\frac{\left(\omega-\omega_{i}\right)^{3}}{D^{6}}\right\}\right|_{\omega=\omega_{i}} \delta\left(\omega-\omega_{i}\right)\right]+\beta_{16} \Theta\left(k^{2}-\omega^{2}\right)
\end{aligned}
$$

where cut parts of the spectral functions are given as 


$$
\begin{aligned}
& \beta_{4}=\frac{1}{\pi} \operatorname{Im}\left(\frac{1}{D^{2}}\right)=-\frac{1}{\pi} \frac{\operatorname{Im} D^{2}}{\left(\operatorname{Re} D^{2}\right)^{2}+\left(\operatorname{Im} D^{2}\right)^{2}}, \\
& \beta_{5}=\frac{1}{\pi} \operatorname{Im}\left(\frac{1}{D^{4}}\right)=-\frac{1}{\pi} \frac{2 \operatorname{Re} D^{2} \operatorname{Im} D^{2}}{\left[\left(\operatorname{Re} D^{2}\right)^{2}+\left(\operatorname{Im} D^{2}\right)^{2}\right]^{2}}, \\
& \beta_{6}=\frac{1}{\pi} \operatorname{Im}\left(\frac{1}{D^{6}}\right)=\frac{1}{\pi} \frac{\left(\operatorname{Im} D^{2}\right)^{3}-3\left(\operatorname{Re} D^{2}\right)^{2} \operatorname{Im} D^{2}}{\left[\left(\operatorname{Re} D^{2}\right)^{2}+\left(\operatorname{Im} D^{2}\right)^{2}\right]^{3}}, \\
& \beta_{7}=\frac{1}{\pi} \operatorname{Im}\left(\frac{b^{\prime}}{D^{2}}\right)=\frac{1}{\pi} \frac{-\operatorname{Re} b^{\prime} \operatorname{Im} D^{2}+\operatorname{Im} b^{\prime} \operatorname{Re} D^{2}}{\left(\operatorname{Re} D^{2}\right)^{2}+\left(\operatorname{Im} D^{2}\right)^{2}}, \\
& \beta_{8}=\frac{1}{\pi} \operatorname{Im}\left(\frac{c^{\prime}}{D^{2}}\right)=\frac{1}{\pi} \frac{-\operatorname{Re} c^{\prime} \operatorname{Im} D^{2}+\operatorname{Im} c^{\prime} \operatorname{Re} D^{2}}{\left(\operatorname{Re} D^{2}\right)^{2}+\left(\operatorname{Im} D^{2}\right)^{2}}, \\
& \beta_{9}^{(0)}=\frac{1}{2} \operatorname{Im}\left(\frac{h(1+a)}{D^{4}}\right)=\frac{1}{\pi} \frac{\operatorname{Im}(h(1+a))\left[\left(\operatorname{Re} D^{2}\right)^{2}-\left(\operatorname{Im} D^{2}\right)^{2}\right]-2 \operatorname{Re} D^{2} \operatorname{Im} D^{2} \operatorname{Re}(h(1+a))}{\left[\left(\operatorname{Re} D^{2}\right)^{2}+\left(\operatorname{Im} D^{2}\right)^{2}\right]^{2}}, \\
& \beta_{9}^{(1)}=\frac{1}{2} \operatorname{Im}\left(\frac{h(1+a) k_{0}}{D^{4}}\right)=\frac{1}{\pi} \frac{\operatorname{Im}\left(h k_{0}(1+a)\right)\left[\left(\operatorname{Re} D^{2}\right)^{2}-\left(\operatorname{Im} D^{2}\right)^{2}\right]-2 \operatorname{Re} D^{2} \operatorname{Im} D^{2} \operatorname{Re}\left(h k_{0}(1+a)\right)}{\left[\left(\operatorname{Re} D^{2}\right)^{2}+\left(\operatorname{Im} D^{2}\right)^{2}\right]^{2}}, \\
& \beta_{10}=\frac{1}{2} \operatorname{Im}\left(\frac{h b}{D^{4}}\right)=\frac{1}{\pi} \frac{\operatorname{Im}(h b)\left[\left(\operatorname{Re} D^{2}\right)^{2}-\left(\operatorname{Im} D^{2}\right)^{2}\right]-2 \operatorname{Re} D^{2} \operatorname{Im} D^{2} \operatorname{Re}(h b)}{\left[\left(\operatorname{Re} D^{2}\right)^{2}+\left(\operatorname{Im} D^{2}\right)^{2}\right]^{2}}, \\
& \beta_{11}=\frac{1}{2} \operatorname{Im}\left(\frac{h b^{\prime}}{D^{4}}\right)=\frac{1}{\pi} \frac{\operatorname{Im}\left(h b^{\prime}\right)\left[\left(\operatorname{Re} D^{2}\right)^{2}-\left(\operatorname{Im} D^{2}\right)^{2}\right]-2 \operatorname{Re} D^{2} \operatorname{Im} D^{2} \operatorname{Re}\left(h b^{\prime}\right)}{\left[\left(\operatorname{Re} D^{2}\right)^{2}+\left(\operatorname{Im} D^{2}\right)^{2}\right]^{2}}, \\
& \beta_{12}=\frac{1}{2} \operatorname{Im}\left(\frac{h c^{\prime}}{D^{4}}\right)=\frac{1}{\pi} \frac{\operatorname{Im}\left(h c^{\prime}\right)\left[\left(\operatorname{Re} D^{2}\right)^{2}-\left(\operatorname{Im} D^{2}\right)^{2}\right]-2 \operatorname{Re} D^{2} \operatorname{Im} D^{2} \operatorname{Re}\left(h c^{\prime}\right)}{\left[\left(\operatorname{Re} D^{2}\right)^{2}+\left(\operatorname{Im} D^{2}\right)^{2}\right]^{2}}, \\
& \beta_{13}=\frac{1}{2} \operatorname{Im}\left(\frac{h^{\prime} b}{D^{4}}\right)=\frac{1}{\pi} \frac{\operatorname{Im}\left(h^{\prime} b\right)\left[\left(\operatorname{Re} D^{2}\right)^{2}-\left(\operatorname{Im} D^{2}\right)^{2}\right]-2 \operatorname{Re} D^{2} \operatorname{Im} D^{2} \operatorname{Re}\left(h^{\prime} b\right)}{\left[\left(\operatorname{Re} D^{2}\right)^{2}+\left(\operatorname{Im} D^{2}\right)^{2}\right]^{2}}, \\
& \beta_{14}^{(0)}=\frac{1}{2} \operatorname{Im}\left(\frac{h^{\prime}(1+a)}{D^{4}}\right)=\frac{1}{\pi} \frac{\operatorname{Im}\left(h^{\prime}(1+a)\right)\left[\left(\operatorname{Re} D^{2}\right)^{2}-\left(\operatorname{Im} D^{2}\right)^{2}\right]-2 \operatorname{Re} D^{2} \operatorname{Im} D^{2} \operatorname{Re}\left(h^{\prime}(1+a)\right)}{\left[\left(\operatorname{Re} D^{2}\right)^{2}+\left(\operatorname{Im} D^{2}\right)^{2}\right]^{2}}, \\
& \beta_{14}^{(1)}=\frac{1}{2} \operatorname{Im}\left(\frac{h^{\prime}(1+a) k_{0}}{D^{4}}\right)=\frac{1}{\pi} \frac{\operatorname{Im}\left(h^{\prime}(1+a) k_{0}\right)\left[\left(\operatorname{Re} D^{2}\right)^{2}-\left(\operatorname{Im} D^{2}\right)^{2}\right]-2 \operatorname{Re} D^{2} \operatorname{Im} D^{2} \operatorname{Re}\left(h^{\prime}(1+a) k_{0}\right)}{\left[\left(\operatorname{Re} D^{2}\right)^{2}+\left(\operatorname{Im} D^{2}\right)^{2}\right]^{2}}, \\
& \beta_{15}^{(0)}=\frac{1}{\pi} \operatorname{Im}\left(\frac{h^{2}(1+a)}{D^{6}}\right)=\frac{1}{\pi} \frac{\operatorname{Im}\left(h^{2}(1+a)\right)\left[\left(\operatorname{Re} D^{2}\right)^{3}-3 \operatorname{Re} D^{2}\left(\operatorname{Im} D^{2}\right)^{2}\right]+\operatorname{Re}\left(h^{2}(1+a)\right)\left[\left(\operatorname{Im} D^{2}\right)^{3}-3 \operatorname{Im} D^{2}\left(\operatorname{Re} D^{2}\right)^{2}\right]}{\left[\left(\operatorname{Re} D^{2}\right)^{2}+\left(\operatorname{Im} D^{2}\right)^{2}\right]^{3}} \\
& \beta_{15}^{(1)}=\frac{1}{\pi} \operatorname{Im}\left(\frac{h^{2}(1+a) k_{0}}{D^{6}}\right)=\frac{1}{\pi} \frac{\operatorname{Im}\left(h^{2}(1+a) k_{0}\right)\left[\left(\operatorname{Re} D^{2}\right)^{3}-3 \operatorname{Re} D^{2}\left(\operatorname{Im} D^{2}\right)^{2}\right]+\operatorname{Re}\left(h^{2}(1+a) k_{0}\right)\left[\left(\operatorname{Im} D^{2}\right)^{3}-3 \operatorname{Im} D^{2}\left(\operatorname{Re} D^{2}\right)^{2}\right]}{\left[\left(\operatorname{Re} D^{2}\right)^{2}+\left(\operatorname{Im} D^{2}\right)^{2}\right]^{3}} \\
& \beta_{16}=\frac{1}{\pi} \operatorname{Im}\left(\frac{h^{2} b}{D^{6}}\right)=\frac{1}{\pi} \frac{\operatorname{Im}\left(h^{2} b\right)\left[\left(\operatorname{Re} D^{2}\right)^{3}-3 \operatorname{Re} D^{2}\left(\operatorname{Im} D^{2}\right)^{2}\right]+\operatorname{Re}\left(h^{2} b\right)\left[\left(\operatorname{Im} D^{2}\right)^{3}-3 \operatorname{Im} D^{2}\left(\operatorname{Re} D^{2}\right)^{2}\right]}{\left[\left(\operatorname{Re} D^{2}\right)^{2}+\left(\operatorname{Im} D^{2}\right)^{2}\right]^{3}}
\end{aligned}
$$

where 


$$
\begin{gathered}
\operatorname{Im}\left(D^{2}\right)=-\frac{\pi m_{\mathrm{th}}^{4}}{k^{2}}\left[\frac{\omega}{k}+\left(1-\frac{\omega^{2}}{k^{2}}\right) Q_{0}\left(\frac{\omega}{k}\right)\right], \\
\operatorname{Re}\left(D^{2}\right)=\omega^{2}-k^{2}-2 m_{\mathrm{th}}^{2}+\frac{m_{\mathrm{th}}^{4}}{k^{2}}\left(\frac{2 \omega}{k} Q_{0}\left(\frac{\omega}{k}\right)-1\right)+\frac{m_{\mathrm{th}}^{4}}{k^{2}}\left(1-\frac{\omega^{2}}{k^{2}}\right)\left(Q_{0}^{2}\left(\frac{\omega}{k}\right)-\frac{\pi^{2}}{4}\right), \\
\operatorname{Im}\left(b^{\prime}\right)=-4 e^{2} M^{2} \frac{\pi k_{3} \omega}{2 k^{3}}, \\
\operatorname{Re}\left(b^{\prime}\right)=4 e^{2} M^{2} \frac{k_{3}}{k^{2}}\left[\frac{\omega}{k} Q_{0}\left(\frac{\omega}{k}\right)-1\right], \\
\operatorname{Im}\left(c^{\prime}\right)=-4 e^{2} M^{2} \frac{\pi}{2 k}, \\
\operatorname{Re}\left(c^{\prime}\right)=4 e^{2} M^{2} \frac{1}{k} Q_{0}\left(\frac{\omega}{k}\right),
\end{gathered}
$$

$$
\begin{aligned}
\operatorname{Im}(h(1+a))= & \frac{4 \pi e^{2} M^{2} k_{3}}{2 k^{7}}\left(-2 k^{6}-2 k^{4}\left(k_{0}^{2}+3 m_{\mathrm{th}}^{2}\right)+12 k^{3} k_{0} m_{\mathrm{th}}^{2} Q_{0}-4 k^{2} m_{\mathrm{th}}^{2}\left(k_{0}^{2}+m_{\mathrm{th}}^{2}\right)\right. \\
& \left.+4 k k_{0} m_{\mathrm{th}}^{2} Q_{0}\left(k_{0}^{2}+4 m_{\mathrm{th}}^{2}\right)+k_{0}^{2} m_{\mathrm{th}}^{4}\left(\pi^{2}-12 Q_{0}^{2}\right)\right) \\
\operatorname{Re}(h(1+a))= & \frac{4 e^{2} M^{2} k_{3}}{2 k^{7}}\left(4 k^{6} Q_{0}-4 k^{5} k_{0}+4 k^{4} Q_{0}\left(k_{0}^{2}+3 m_{\mathrm{th}}^{2}\right)+k^{3} k_{0} m_{\mathrm{th}}^{2}\left(-12 Q_{0}^{2}+3 \pi^{2}-4\right)\right. \\
& \left.+8 k^{2} m_{\mathrm{th}}^{2} Q_{0}\left(k_{0}^{2}+m_{\mathrm{th}}^{2}\right)+k k_{0} m_{\mathrm{th}}^{2}\left(\pi^{2}-4 Q_{0}^{2}\right)\left(k_{0}^{2}+4 m_{\mathrm{th}}^{2}\right)+2 k_{0}^{2} m_{\mathrm{th}}^{4} Q_{0}\left(4 Q_{0}^{2}-3 \pi^{2}\right)\right), \\
\operatorname{Im}(h b)= & \frac{4 \pi e^{2} M^{2} k_{3} m_{\mathrm{th}}^{2}}{2 k^{7}}\left(4 k^{2} k_{0}\left(k_{0}^{2}+m_{\mathrm{th}}^{2}\right)+4 k Q_{0}\left(k^{4}+2 m_{\mathrm{th}}^{2}\left(k^{2}-2 k_{0}^{2}\right)-k_{0}^{4}\right)\right. \\
& \left.+12 k_{0} m_{\mathrm{th}}^{2} Q_{0}^{2}\left(k_{0}-k\right)\left(k+k_{0}\right)+\pi^{2} k_{0} m_{\mathrm{th}}^{2}\left(k-k_{0}\right)\left(k+k_{0}\right)\right),
\end{aligned}
$$

$$
\begin{aligned}
\operatorname{Re}(h b)= & \frac{4 e^{2} M^{2} k_{3} m_{\mathrm{th}}^{2}}{2 k^{7}}\left(k^{5}\left(\pi^{2}-4 Q_{0}^{2}\right)+2 k^{3}\left(2 k_{0}^{2}+m_{\mathrm{th}}^{2}\left(\pi^{2}-4 Q_{0}^{2}\right)\right)-2 k^{2} k_{0} Q_{0}\left(4 k_{0}^{2}+m_{\mathrm{th}}^{2}\left(-4 Q_{0}^{2}+3 \pi^{2}+4\right)\right)\right. \\
& \left.-k k_{0}^{2}\left(\pi^{2}-4 Q_{0}^{2}\right)\left(k_{0}^{2}+4 m_{\mathrm{th}}^{2}\right)+2 k_{0}^{3} m_{\mathrm{th}}^{2} Q_{0}\left(3 \pi^{2}-4 Q_{0}^{2}\right)\right), \\
& \operatorname{Im}\left(h b^{\prime}\right)=\frac{16 \pi e^{4} M^{4} k_{3}^{2}}{2 k^{7}}\left(2 k^{4}-4 k^{3} k_{0} Q_{0}+4 k^{2}\left(k_{0}^{2}+m_{\mathrm{th}}^{2}\right)-4 k k_{0} Q_{0}\left(k_{0}^{2}+4 m_{\mathrm{th}}^{2}\right)-k_{0}^{2} m_{\mathrm{th}}^{2}\left(\pi^{2}-12 Q_{0}^{2}\right)\right),
\end{aligned}
$$

$$
\begin{aligned}
\operatorname{Re}\left(h b^{\prime}\right)= & -\frac{16 e^{4} M^{4} k_{3}^{2}}{2 k^{7}}\left(4 k^{4} Q_{0}+k^{3} k_{0}\left(\pi^{2}-4\left(Q_{0}^{2}+1\right)\right)+8 k^{2} Q_{0}\left(k_{0}^{2}+m_{\mathrm{th}}^{2}\right)+k k_{0}\left(\pi^{2}-4 Q_{0}^{2}\right)\left(k_{0}^{2}+4 m_{\mathrm{th}}^{2}\right)\right. \\
+ & \left.2 k_{0}^{2} m_{\mathrm{th}}^{2} Q_{0}\left(4 Q_{0}^{2}-3 \pi^{2}\right)\right) \\
& \operatorname{Im}\left(h c^{\prime}\right)=-\frac{16 \pi e^{4} M^{4} k_{3}}{2 k^{5}}\left(4 k^{3} Q_{0}-2 k^{2} k_{0}+4 k Q_{0}\left(k_{0}^{2}+2 m_{\mathrm{th}}^{2}\right)+k_{0} m_{\mathrm{th}}^{2}\left(\pi^{2}-12 Q_{0}^{2}\right)\right)
\end{aligned}
$$$$
\operatorname{Re}\left(h c^{\prime}\right)=-\frac{16 e^{4} M^{4} k_{3}}{2 k^{5}}\left(k^{3}\left(\pi^{2}-4 Q_{0}^{2}\right)+4 k^{2} k_{0} Q_{0}+k\left(\pi^{2}-4 Q_{0}^{2}\right)\left(k_{0}^{2}+2 m_{\mathrm{th}}^{2}\right)+2 k_{0} m_{\mathrm{th}}^{2} Q_{0}\left(4 Q_{0}^{2}-3 \pi^{2}\right)\right)
$$

$$
\begin{aligned}
\operatorname{Im}\left(h^{\prime} b\right)= & \frac{8 \pi e^{4} M^{4} m_{\mathrm{th}}^{2}}{k^{9}}\left(k^{6}\left(\frac{\pi^{2}}{4}-3 Q_{0}^{2}\right)-2 k^{5} \omega Q_{0}+k^{4}\left(k_{3}^{2}-\omega^{2}\left(\frac{\pi^{2}}{4}-3 Q_{0}^{2}\right)\right)\right. \\
& \left.-4 k^{3} \omega k_{3}^{2} Q_{0}-k^{2} \omega^{2} k_{3}^{2}\left(-3 Q_{0}^{2}+\frac{\pi^{2}}{4}+3\right)+6 k \omega^{3} k_{3}^{2} Q_{0}+\omega^{4} k_{3}^{2}\left(\frac{\pi^{2}}{4}-3 Q_{0}^{2}\right)\right),
\end{aligned}
$$




$$
\begin{aligned}
\operatorname{Re}\left(h^{\prime} b\right)= & \frac{16 e^{4} M^{4} m_{\mathrm{th}}^{2}}{k^{9}}\left(k^{6}\left(Q_{0}^{3}-\frac{3 \pi^{2}}{4} Q_{0}\right)+k^{5} \omega\left(Q_{0}^{2}-\frac{\pi^{2}}{4}\right)-k^{4} Q_{0}\left(\omega^{2}\left(Q_{0}^{2}-\frac{3 \pi^{2}}{4}\right)+k_{3}^{2}\right)+k^{3} \omega k_{3}^{2}\left(2 Q_{0}^{2}-\frac{\pi^{2}}{2}-1\right)\right. \\
& \left.+k^{2} \omega^{2} k_{3}^{2} Q_{0}\left(-Q_{0}^{2}+\frac{3 \pi^{2}}{4}+3\right)+3 k \omega^{3} k_{3}^{2}\left(\frac{\pi^{2}}{4}-Q_{0}^{2}\right)+\omega^{4} k_{3}^{2} Q_{0}\left(Q_{0}^{2}-\frac{3 \pi^{2}}{4}\right)\right),
\end{aligned}
$$

$$
\begin{aligned}
\operatorname{Im}\left(h^{\prime}(1+a)\right)= & \frac{8 \pi e^{4} M^{4}}{k^{9}}\left(2 k^{7} Q_{0}+2 k^{5} m_{\mathrm{th}}^{2} Q_{0}+k^{4} \omega\left(2 k_{3}^{2}+m_{\mathrm{th}}^{2}\left(\frac{\pi^{2}}{4}-3 Q_{0}^{2}\right)\right)-2 k^{3} \omega^{2} k_{3}^{2} Q_{0}\right. \\
& \left.+3 k^{2} \omega k_{3}^{2} m_{\mathrm{th}}^{2}-6 k \omega^{2} k_{3}^{2} m_{\mathrm{th}}^{2} Q_{0}-\omega^{3} k_{3}^{2} m_{\mathrm{th}}^{2}\left(\frac{\pi^{2}}{4}-3 Q_{0}^{2}\right)\right),
\end{aligned}
$$

$$
\begin{aligned}
\operatorname{Re}\left(h^{\prime}(1+a)\right)= & \frac{16 e^{4} M^{4}}{k^{9}}\left(k^{7}\left(\frac{\pi^{2}}{4}-Q_{0}^{2}\right)+k^{5}\left(k_{3}^{2}+m_{\mathrm{th}}^{2}\left(\frac{\pi^{2}}{4}-Q_{0}^{2}\right)\right)+k^{4} \omega Q_{0}\left(m_{\mathrm{th}}^{2}\left(Q_{0}^{2}-\frac{3 \pi^{2}}{4}\right)-2 k_{3}^{2}\right)\right. \\
& \left.+k^{3} k_{3}^{2}\left(\omega^{2}\left(Q_{0}^{2}-\frac{\pi^{2}}{4}\right)+m_{\mathrm{th}}^{2}\right)-3 k^{2} \omega k_{3}^{2} m_{\mathrm{th}}^{2} Q_{0}+3 k \omega^{2} k_{3}^{2} m_{\mathrm{th}}^{2}\left(Q_{0}^{2}-\frac{\pi^{2}}{4}\right)-\omega^{3} k_{3}^{2} m_{\mathrm{th}}^{2} Q_{0}\left(Q_{0}^{2}-\frac{3 \pi^{2}}{4}\right)\right)
\end{aligned}
$$

$$
\begin{aligned}
\operatorname{Im}\left(h^{2}(1+a)\right)= & \frac{16 \pi e^{4} M^{4} k_{3}^{2}}{2 k^{11}}\left(-8 k^{9} Q_{0}+8 k^{8} k_{0}-8 k^{7} Q_{0}\left(2 k_{0}^{2}+5 m_{\mathrm{th}}^{2}\right)+k^{6} k_{0}\left(8 k_{0}^{2}+m_{\mathrm{th}}^{2}\left(60 Q_{0}^{2}-5 \pi^{2}+24\right)\right)\right. \\
& -8 k^{5} Q_{0}\left(k_{0}^{4}+12 k_{0}^{2} m_{\mathrm{th}}^{2}+8 m_{\mathrm{th}}^{4}\right)+2 k^{4} k_{0} m_{\mathrm{th}}^{2}\left(k_{0}^{2}\left(36 Q_{0}^{2}-3 \pi^{2}+6\right)-8 m_{\mathrm{th}}^{2}\left(-12 Q_{0}^{2}+\pi^{2}-1\right)\right) \\
& -8 k^{3} m_{\mathrm{th}}^{2} Q_{0}\left(3 k_{0}^{4}-4 k_{0}^{2} m_{\mathrm{th}}^{2}\left(-4 Q_{0}^{2}+\pi^{2}-3\right)+4 m_{\mathrm{th}}^{4}\right)-k^{2} k_{0} m_{\mathrm{th}}^{2}\left(\pi^{2}-12 Q_{0}^{2}\right)\left(k_{0}^{4}+12 k_{0}^{2} m_{\mathrm{th}}^{2}\right. \\
& \left.\left.+12 m_{\mathrm{th}}^{4}\right)+16 k k_{0}^{2} m_{\mathrm{th}}^{4} Q_{0}\left(\pi^{2}-4 Q_{0}^{2}\right)\left(k_{0}^{2}+3 m_{\mathrm{th}}^{2}\right)+k_{0}^{3} m_{\mathrm{th}}^{6}\left(80 Q_{0}^{4}-40 \pi^{2} Q_{0}^{2}+\pi^{4}\right)\right),
\end{aligned}
$$

$$
\begin{aligned}
\operatorname{Re}\left(h^{2}(1+a)\right)= & -\frac{16 e^{4} M^{4} k_{3}^{2}}{k^{11}}\left(k^{9}\left(\pi^{2}-4 Q_{0}^{2}\right)+8 k^{8} k_{0} Q_{0}+k^{7}\left(2 k_{0}^{2}\left(-4 Q_{0}^{2}+\pi^{2}-2\right)+5 m_{\mathrm{th}}^{2}\left(\pi^{2}-4 Q_{0}^{2}\right)\right)\right. \\
& +k^{6} k_{0} Q_{0}\left(8 k_{0}^{2}+m_{\mathrm{th}}^{2}\left(20 Q_{0}^{2}-15 \pi^{2}+24\right)\right)+k^{5}\left(k_{0}^{4}\left(\pi^{2}-4 Q_{0}^{2}\right)+4 k_{0}^{2} m_{\mathrm{th}}^{2}\left(-12 Q_{0}^{2}+3 \pi^{2}-1\right)+8 m_{\mathrm{th}}^{4}\left(\pi^{2}-4 Q_{0}^{2}\right)\right) \\
& +2 k^{4} k_{0} m_{\mathrm{th}}^{2} Q_{0}\left(k_{0}^{2}\left(12 Q_{0}^{2}-9 \pi^{2}+6\right)+8 m_{\mathrm{th}}^{2}\left(4 Q_{0}^{2}-3 \pi^{2}+1\right)\right) \\
& +k^{3} m_{\mathrm{th}}^{2}\left(3 k_{0}^{4}\left(\pi^{2}-4 Q_{0}^{2}\right)-2 k_{0}^{2} m_{\mathrm{th}}^{2}\left(8\left(2 Q_{0}^{2}+3\right) Q_{0}^{2}-6 \pi^{2}\left(4 Q_{0}^{2}+1\right)+\pi^{4}\right)\right. \\
& \left.+4 m_{\mathrm{th}}^{4}\left(\pi^{2}-4 Q_{0}^{2}\right)\right)+k^{2} k_{0} m_{\mathrm{th}}^{2} Q_{0}\left(4 Q_{0}^{2}-3 \pi^{2}\right)\left(k_{0}^{4}+12 k_{0}^{2} m_{\mathrm{th}}^{2}+12 m_{\mathrm{th}}^{4}\right) \\
& \left.-k k_{0}^{2} m_{\mathrm{th}}^{4}\left(16 Q_{0}^{4}-24 \pi^{2} Q_{0}^{2}+\pi^{4}\right)\left(k_{0}^{2}+3 m_{\mathrm{th}}^{2}\right)+k_{0}^{3} m_{\mathrm{th}}^{6} Q_{0}\left(16 Q_{0}^{4}-40 \pi^{2} Q_{0}^{2}+5 \pi^{4}\right)\right)
\end{aligned}
$$

$$
\begin{aligned}
\operatorname{Im}\left(h^{2} b\right)= & \frac{16 \pi e^{4} M^{4} k_{3}^{2} m_{\mathrm{th}}^{2}}{2 k^{11}}\left(k^{8}\left(-\left(\pi^{2}-12 Q_{0}^{2}\right)\right)-8 k^{7} k_{0} Q_{0}-k^{6}\left(k_{0}^{2}\left(-12 Q_{0}^{2}+\pi^{2}+4\right)+4 m_{\mathrm{th}}^{2}\left(\pi^{2}-12 Q_{0}^{2}\right)\right)\right. \\
& +16 k^{5} k_{0} Q_{0}\left(k_{0}^{2}+m_{\mathrm{th}}^{2}\left(\pi^{2}-4 Q_{0}^{2}\right)\right)+k^{4}\left(k_{0}^{4}\left(\pi^{2}-12\left(Q_{0}^{2}+1\right)\right)-16 k_{0}^{2} m_{\mathrm{th}}^{2}-4 m_{\mathrm{th}}^{4}\left(\pi^{2}-12 Q_{0}^{2}\right)\right) \\
& +8 k^{3} k_{0} Q_{0}\left(3 k_{0}^{4}+12 k_{0}^{2} m_{\mathrm{th}}^{2}+4 m_{\mathrm{th}}^{4}\left(-4 Q_{0}^{2}+\pi^{2}+1\right)\right)+k^{2} k_{0}^{2}\left(k_{0}^{4}\left(\pi^{2}-12 Q_{0}^{2}\right)+12 k_{0}^{2} m_{\mathrm{th}}^{2}\left(\pi^{2}-12 Q_{0}^{2}\right)\right. \\
& \left.+m_{\mathrm{th}}^{4}\left(80 Q_{0}^{4}-8\left(18+5 \pi^{2}\right) Q_{0}^{2}+\pi^{2}\left(12+\pi^{2}\right)\right)\right)-16 k k_{0}^{3} m_{\mathrm{th}}^{2} Q_{0}\left(\pi^{2}-4 Q_{0}^{2}\right)\left(k_{0}^{2}+3 m_{\mathrm{th}}^{2}\right) \\
& \left.-k_{0}^{4} m_{\mathrm{th}}^{4}\left(80 Q_{0}^{4}-40 \pi^{2} Q_{0}^{2}+\pi^{4}\right)\right),
\end{aligned}
$$




$$
\begin{aligned}
\operatorname{Re}\left(h^{2} b\right)= & \frac{16 e^{4} M^{4} k_{3}^{2} m_{\mathrm{th}}^{2}}{k^{11}}\left(k^{8}\left(3 \pi^{2} Q_{0}-4 Q_{0}^{3}\right)-k^{7} k_{0}\left(\pi^{2}-4 Q_{0}^{2}\right)+k^{6} Q_{0}\left(k_{0}^{2}\left(-4 Q_{0}^{2}+3 \pi^{2}+4\right)\right.\right. \\
& \left.+4 m_{\mathrm{th}}^{2}\left(3 \pi^{2}-4 Q_{0}^{2}\right)\right)+k^{5} k_{0}\left(2 k_{0}^{2}\left(-4 Q_{0}^{2}+\pi^{2}-2\right)+m_{\mathrm{th}}^{2}\left(16 Q_{0}^{4}-24 \pi^{2} Q_{0}^{2}+\pi^{4}\right)\right) \\
& +k^{4} Q_{0}\left(k_{0}^{4}\left(4 Q_{0}^{2}-3 \pi^{2}+12\right)+16 k_{0}^{2} m_{\mathrm{th}}^{2}+4 m_{\mathrm{th}}^{4}\left(3 \pi^{2}-4 Q_{0}^{2}\right)\right)+k^{3} k_{0}\left(3 k_{0}^{4}\left(\pi^{2}-4 Q_{0}^{2}\right)\right. \\
& \left.+12 k_{0}^{2} m_{\mathrm{th}}^{2}\left(\pi^{2}-4 Q_{0}^{2}\right)+2 m_{\mathrm{th}}^{4}\left(16 Q_{0}^{4}-8\left(1+3 \pi^{2}\right) Q_{0}^{2}+\pi^{2}\left(2+\pi^{2}\right)\right)\right)+k^{2} k_{0}^{2} Q_{0} \\
& \times\left(4 Q_{0}^{2}\left(k_{0}^{4}+12 k_{0}^{2} m_{\mathrm{th}}^{2}+2\left(6+5 \pi^{2}\right) m_{\mathrm{th}}^{4}\right)-\pi^{2}\left(3 k_{0}^{4}+36 k_{0}^{2} m_{\mathrm{th}}^{2}+\left(36+5 \pi^{2}\right) m_{\mathrm{th}}^{4}\right)-16 m_{\mathrm{th}}^{4} Q_{0}^{4}\right) \\
& \left.-k k_{0}^{3} m_{\mathrm{th}}^{2}\left(16 Q_{0}^{4}-24 \pi^{2} Q_{0}^{2}+\pi^{4}\right)\left(k_{0}^{2}+3 m_{\mathrm{th}}^{2}\right)+k_{0}^{4} m_{\mathrm{th}}^{4} Q_{0}\left(16 Q_{0}^{4}-40 \pi^{2} Q_{0}^{2}+5 \pi^{4}\right)\right),
\end{aligned}
$$

where $M^{2}$ is defined in Eq. (29).

[1] D. A. Uzdensky and S. Rightley, Plasma physics of extreme astrophysical environments, Rep. Prog. Phys. 77, 036902 (2014).

[2] A. K. Ganguly, S. Konar, and P. B. Pal, Faraday effect: A field theoretical point of view, Phys. Rev. D 60, 105014 (1999).

[3] M. Giovannini, Cosmic microwave background polarization, Faraday rotation, and stochastic gravity-waves backgrounds, Phys. Rev. D 56, 3198 (1997).

[4] E. P. Liang, S. C. Wilks, and M Tabak, Pair production by ultraintense Lasers, Phys. Rev. Lett. 81, 4887 (1998).

[5] M. H. Thoma, Field theoretic description of ultrarelativistic electron-positron plasmas, Rev. Mod. Phys. 81, 959 (2009).

[6] M. H. Thoma, What can we learn from electromagnetic plasmas about the quark-gluon plasma?, J. Phys. A 42, 214004 (2009).

[7] V. Skokov, A. Y. Illarionov, and V. Toneev, Estimate of the magnetic field strength in heavy-ion collisions, Int. J. Mod. Phys. A 24, 5925 (2009).

[8] A. Bzdak and V. Skokov, Anisotropy of Photon Production: Initial Eccentricity or Magnetic Field, Phys. Rev. Lett. 110, 192301 (2013).

[9] L. McLerran and V. Skokov, Comments about the electromagnetic field in heavy-ion collisions, Nucl. Phys. A929, 184 (2014).

[10] K. Tuchin, Electromagnetic radiation by quark-gluon plasma in a magnetic field, Phys. Rev. C 87, 024912 (2013).

[11] K. Tuchin, Magnetic contribution to dilepton production in heavy-ion collisions, Phys. Rev. C 88, 024910 (2013).

[12] K. Tuchin, Particle production in strong electromagnetic fields in relativistic heavy-ion collisions, Adv. High Energy Phys. 2013, 1 (2013).

[13] J. Alexandre, K. Farakos, and G. Koutsoumbas, Magnetic catalysis in QED(3) at finite temperature: Beyond the constant mass approximation, Phys. Rev. D 63, 065015 (2001).

[14] V. P. Gusynin and I. A. Shovkovy, Chiral symmetry breaking in QED in a magnetic field at finite temperature, Phys. Rev. D 56, 5251 (1997).

[15] D. S. Lee, C. N. Leung, and Y. J. Ng, Chiral symmetry breaking in a uniform external magnetic field, Phys. Rev. D 55, 6504 (1997).
[16] V. P. Gusynin, V. A. Miransky, and I. A. Shovkovy, Catalysis of Dynamical Flavor Symmetry Breaking by a Magnetic Field in (2+1)-Dimensions, Phys. Rev. Lett. 73, 3499 (1994); Erratum, Phys. Rev. Lett. 76, 1005 (1996).

[17] G. S. Bali, F. Bruckmann, G. Endrodi, Z. Fodor, S. D. Katz, S. Krieg, A. Schafer, and K. K. Szabo, The QCD phase diagram for external magnetic fields, J. High Energy Phys. 02 (2012) 044.

[18] V. G. Bornyakov, P. V. Buividovich, N. Cundy, O. A. Kochetkov, and A. Schäfer, Deconfinement transition in two-flavor lattice QCD with dynamical overlap fermions in an external magnetic field, Phys. Rev. D 90, 034501 (2014).

[19] N. Mueller and J. M. Pawlowski, Magnetic catalysis and inverse magnetic catalysis in QCD, Phys. Rev. D 91, 116010 (2015).

[20] A. Ayala, M. Loewe, A. Z. Mizher, and R. Zamora, Inverse magnetic catalysis for the chiral transition induced by thermo-magnetic effects on the coupling constant, Phys. Rev. D 90, 036001 (2014).

[21] R. L. S. Farias, K. P. Gomes, G. I. Krein, and M. B. Pinto, Importance of asymptotic freedom for the pseudocritical temperature in magnetized quark matter, Phys. Rev. C 90, 025203 (2014).

[22] A. Ayala, M. Loewe, and R. Zamora, Inverse magnetic catalysis in the linear sigma model with quarks, Phys. Rev. D 91, 016002 (2015).

[23] A. Ayala, M. Loewe, and R. Zamora, Inverse magnetic catalysis in the linear sigma model, J. Phys. Conf. Ser. 720, 012026 (2016).

[24] A. Ayala, C. A. Dominguez, L. A. Hernandez, M. Loewe, and R. Zamora, Inverse magnetic catalysis from the properties of the QCD coupling in a magnetic field, Phys. Lett. B 759, 99 (2016).

[25] R. L. S. Farias, V. S. Timoteo, S. S. Avancini, M. B. Pinto, and G. Krein, Thermo-magnetic effects in quark matter: Nambu-Jona-Lasinio model constrained by lattice QCD, Eur. Phys. J. A 53, 101 (2017).

[26] A. Bandyopadhyay, B. Karmakar, N. Haque, and M. G. Mustafa, Pressure of a weakly magnetized hot and dense deconfined QCD matter in one-loop hard-thermal-loop perturbation theory, Phys. Rev. D 100, 034031 (2019). 
[27] B. Karmakar, R. Ghosh, A. Bandyopadhyay, N. Haque, and M. G. Mustafa, Anisotropic pressure of deconfined QCD matter in presence of strong magnetic field within one-loop approximation, Phys. Rev. D 99, 094002 (2019).

[28] S. Rath and B. K. Patra, One-loop QCD thermodynamics in a strong homogeneous and static magnetic field, J. High Energy Phys. 12 (2017) 098.

[29] M. Kurian, S. Mitra, and V. Chandra, Transport coefficients of hot magnetized QCD matter beyond the lowest Landau level approximation, Eur. Phys. J. C 79, 134 (2019).

[30] M. Kurian and V. Chandra, Bulk viscosity of a hot QCD/QGP medium in strong magnetic field within relaxation-time approximation, Phys. Rev. D 97, 116008 (2018).

[31] M. Kurian and V. Chandra, Effective description of hot QCD medium in strong magnetic field and longitudinal conductivity, Phys. Rev. D 96, 114026 (2017).

[32] A. Das, A. Bandyopadhyay, P. K. Roy, and M. G. Mustafa, General structure of fermion two-point function and its spectral representation in a hot magnetized medium, Phys. Rev. D 97, 034024 (2018).

[33] B. Karmakar, A. Bandyopadhyay, N. Haque, and M. G. Mustafa, General structure of gauge boson propagator and its spectra in a hot magnetized medium, Eur. Phys. J. C 79, 658 (2019).

[34] K. Hattori and D. Satow, Gluon spectrum in a quark-gluon plasma under strong magnetic fields, Phys. Rev. D 97, 014023 (2018).

[35] A. Ayala, C. A. Dominguez, S. Hernandez-Ortiz, L. A. Hernandez, M. Loewe, D. Manreza Paret, and R. Zamora, Gluon polarization tensor in a thermo-magnetic medium, arXiv:1805.07344.

[36] A. Das, N. Haque, M. G. Mustafa, and P. K. Roy, Hard dilepton production from a weakly magnetized hot QCD medium, Phys. Rev. D 99, 094022 (2019).

[37] K. Tuchin, Electromagnetic radiation by quark-gluon plasma in a magnetic field, Phys. Rev. C 87, 024912 (2013).
[38] A. Bandyopadhyay, C. A. Islam, and M. G. Mustafa, Electromagnetic spectral properties and Debye screening of a strongly magnetized hot medium, Phys. Rev. D 94, 114034 (2016).

[39] N. Sadooghi and F. Taghinavaz, Dilepton production rate in a hot and magnetized quark-gluon plasma, Ann. Phys. (Amsterdam) 376, 218 (2017).

[40] K. Tuchin, Magnetic contribution to dilepton production in heavy-ion collisions, Phys. Rev. C 88, 024910 (2013).

[41] A. Bandyopadhyay and S. Mallik, Effect of magnetic field on dilepton production in a hot plasma, Phys. Rev. D 95, 074019 (2017).

[42] K. A. Mamo, Enhanced thermal photon and dilepton production in strongly coupled $\mathcal{N}=4 \mathrm{SYM}$ plasma in strong magnetic field, J. High Energy Phys. 08 (2013) 083.

[43] M. H. Thoma, Parton interaction rates in the quark-gluon plasma, Phys. Rev. D 49, 451 (1994).

[44] J. I. Kapusta, P. Lichard, and D. Seibert, High-energy photons from quark-gluon plasma versus hot hadronic gas, Phys. Rev. D 44, 2774 (1991); Erratum, Phys. Rev. D 47, 4171 (1993).

[45] M. H. Thoma, Damping rate of a hard photon in a relativistic plasma, Phys. Rev. D 51, 862 (1995).

[46] H. A. Weldon, Simple rules for discontinuities in finite temperature field theory, Phys. Rev. D 28, 2007 (1983).

[47] E. Braaten, R. D. Pisarski, and T. C. Yuan, Production of Soft Dileptons in the Quark-Gluon Plasma, Phys. Rev. Lett. 64, 2242 (1990).

[48] M.H. Thoma, New developments and applications of thermal field theory, arXiv:hep-ph/0010164.

[49] T. K. Chyi, C. W. Hwang, W. F. Kao, G. L. Lin, K. W. Ng, and J. J. Tseng, The weak field expansion for processes in a homogeneous background magnetic field, Phys. Rev. D 62 , 105014 (2000).

[50] M. L. Bellac, Thermal Field Theory: Cambridge Monographs on Mathematical Physics (Cambridge University Press, Cambridge, England, 1996).

[51] J. I. Kapusta and C. Gale, Finite-Temperature Field Theory: Principles and Applications (Cambridge University Press, Cambridge, England, 2006). 